\title{
WestVirginiaUniversity
}

THE RESEARCH REPOSITORY @ WVU

Graduate Theses, Dissertations, and Problem Reports

2013

\section{Is postural stability compromised in women with urinary incontinence?}

\author{
Samantha K. Rondini \\ West Virginia University
}

Follow this and additional works at: https://researchrepository.wvu.edu/etd

\section{Recommended Citation}

Rondini, Samantha K., "Is postural stability compromised in women with urinary incontinence?" (2013). Graduate Theses, Dissertations, and Problem Reports. 3617.

https://researchrepository.wvu.edu/etd/3617

This Thesis is protected by copyright and/or related rights. It has been brought to you by the The Research Repository @ WVU with permission from the rights-holder(s). You are free to use this Thesis in any way that is permitted by the copyright and related rights legislation that applies to your use. For other uses you must obtain permission from the rights-holder(s) directly, unless additional rights are indicated by a Creative Commons license in the record and/ or on the work itself. This Thesis has been accepted for inclusion in WVU Graduate Theses, Dissertations, and Problem Reports collection by an authorized administrator of The Research Repository @ WVU. For more information, please contact researchrepository@mail.wvu.edu. 


\title{
IS POSTURAL STABILITY COMPROMISED IN WOMEN WITH URINARY INCONTINENCE?
}

\author{
Samantha K. Rondini \\ Thesis submitted to the \\ School of Medicine \\ at West Virginia University \\ in partial fulfillment of the requirements \\ for the degree of \\ Master of Science \\ in \\ Exercise Physiology
}

\author{
Approved by \\ Jean McCrory, Ph.D., Committee Chairperson \\ Corrie Mancinelli, PT, Ph.D. \\ Krystal Thomas, MS, DPT, PT \\ Sergiy Yakovenko, Ph.D.
}

\section{Department of Exercise Physiology}

Morgantown, West Virginia

2013

Keywords: Urinary incontinence, dynamic/static postural stability, balance, center of
pressure
Copyright 2013 Samantha Rondini 


\section{ABSTRACT \\ Is Postural Stability Compromised in Women with Urinary Incontinence?}

\section{Samantha K. Rondini}

Women with urinary incontinence $(\mathrm{UI})$ have an increased risk of falling compared to their age-matched peers without UI. Due to the anatomical location of the pelvic floor muscles, they are suspected to contribute to the maintenance of postural stability during everyday activities. Women with UI have weak or dysfunctional pelvic floor muscles. The purpose of this study was to determine if women with $\mathrm{UI}$, specifically stress, urge, and mixed UI, have deficits in postural stability during static tasks and in response to postural perturbations of the support surface.

Methods: To measure static stability, 14 women ( 7 with UI: age $51.75 \pm 17.85 \mathrm{yrs,}$ height $164.94 \pm 5.40 \mathrm{~cm}$, mass $79.38 \pm 19.45 \mathrm{~kg}$, number of falls $0.714 \pm 1.89$, number of pregnancies $1.29 \pm 0.76$ and 7 controls: age $51.71 \pm 18.20 \mathrm{yrs}$, height $163.29 \pm 6.64$ $\mathrm{cm}$, mass $60.47 \pm 8.32 \mathrm{~kg}$, number of falls $0.00 \pm 0.00$, number of pregnancies $1.29 \pm$ $0.76)$ stood quietly on a force plate with their eyes open and then eyes closed. These same women had their dynamic stability measured by undergoing perturbations of the force plate in both the toes up and toes down directions. A two-factor MANOVA (group $x$ eyes open/closed) was performed on the following static variables: anterioposterior sway, mediolateral sway, length of the path of the center of pressure (COP), sway velocity in quiet stance, and elliptical sway area. Another two-factor MANOVA (group $x$ toes up/down) was performed on the following dynamic variables: initial sway, total sway, and sway velocity. The alpha value for all statistical analyses was 0.05 .

Results: No differences in the static stability variables of anterioposterior sway, mediolateral sway, length of the path of COP, sway velocity in quiet stance, and elliptical sway area were seen between groups. Results of the dynamic stability assessment revealed that the UI group had significantly less initial sway $(p=0.006)$, total sway $(p=0.008)$, and sway velocity $(p=0.001)$ compared to the controls.

Conclusion: Women with UI have less COP movement in response to postural perturbations compared to an age and parity-matched control group. Further studies are needed to determine muscular compensations and co-contractions that may be contributing to this response. 


\section{Table of Contents}

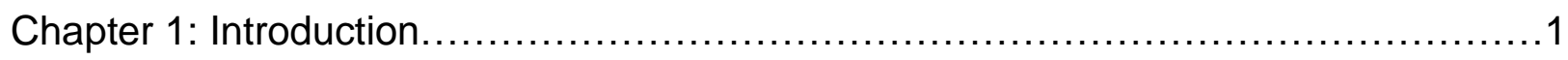

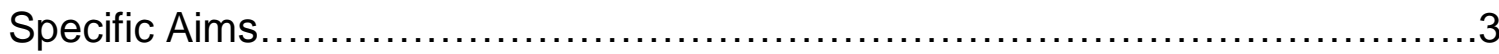

Background and Significance ...............................................

Postural Stability and Balance........................................ 5

Urinary Incontinence.................................................

Significance ................................................................

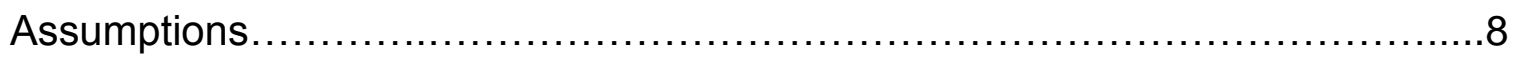

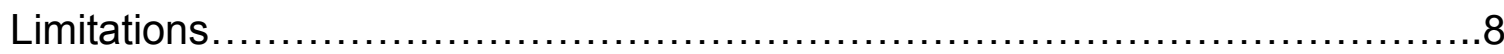

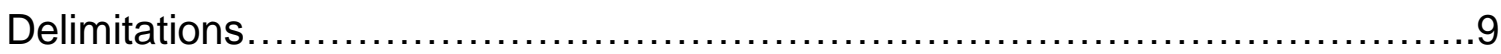

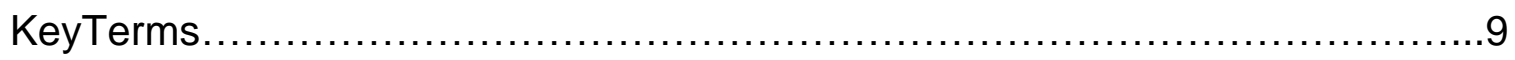

Chapter 2: Literature Review..................................................... 12

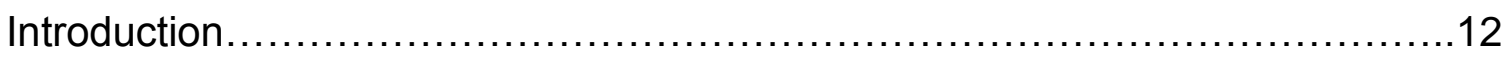

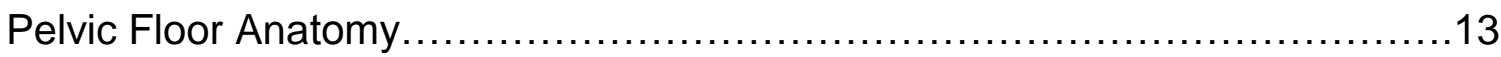

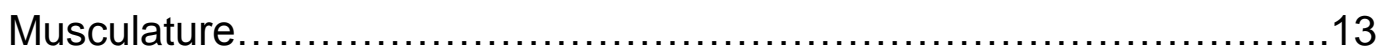

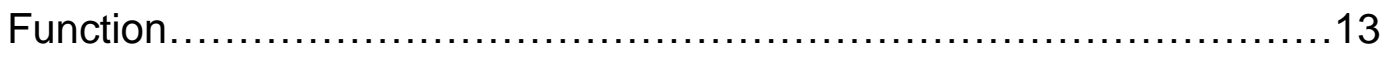

Specific Pelvic Floor Dysfunctions ............................................ 14

General Information................................................ 14

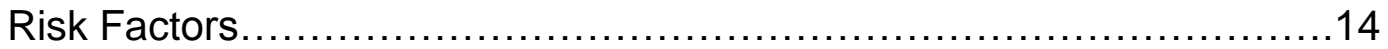

Fecal Incontinence ....................................................

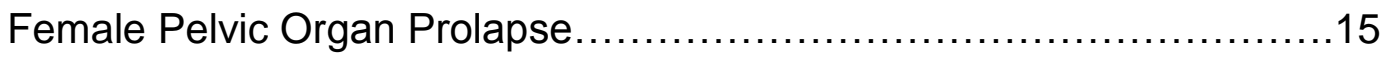

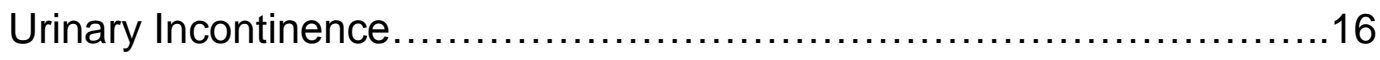

Urinary Incontinence and Muscle Activity ...............................19 
Urinary Incontinence and Postural Stability...................................20

Postural Stability Measurement Techniques....................................21

Clinical Assessments ...............................................22

Static Postural Stability Assessment...................................23

Dynamic Postural Stability Assessment..................................25

Chapter 3: Research Methods and Procedures............................................

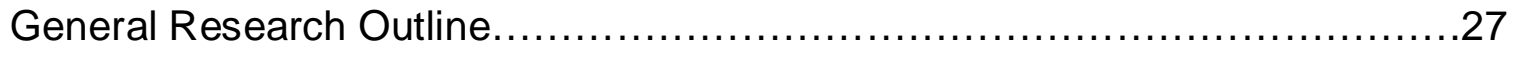

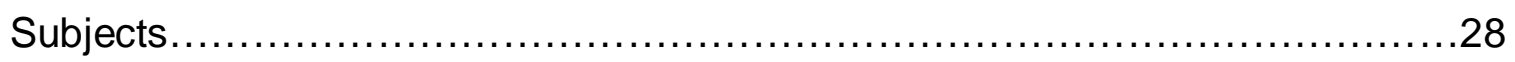

Subject Inclusion/Exclusion Criteria...................................29

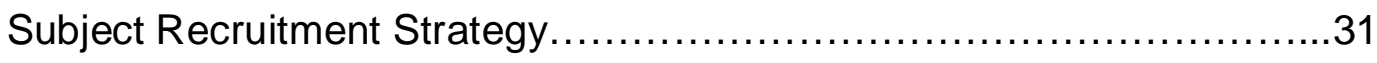

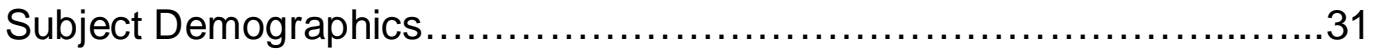

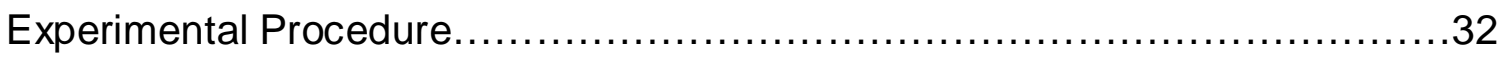

General Health Phone Screening Questionnaire....................32

Informed Consent................................................

Balance and Falls Questionnaire...................................33

Urinary Incontinence Questionnaire ..............................33

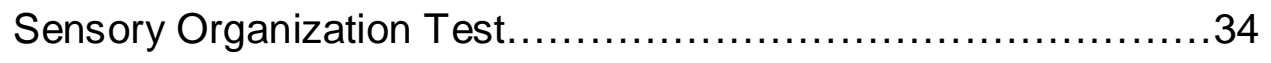

Adaptation Test..................................................

Post-Testing Urinary Incontinence Questionnaire...................40

Statistical Analyses............................................... 40

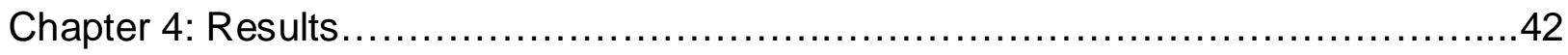

Specific Aim 1: Static Postural Stability.............................42

Specific Aim 2: Dynamic Postural Stability ..........................44 


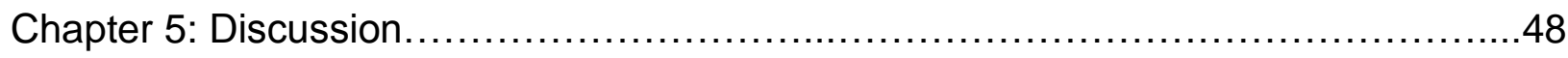

Specific Aim 1: Static Postural Stability............................48

Specific Aim 2: Dynamic Postural Stability .........................52

Limitations........................................................ 57

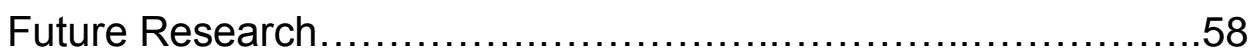

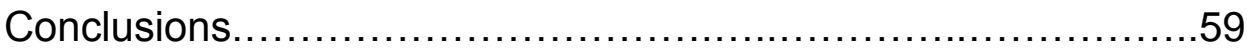

Appendix A: General Health Phone Screening Questionnaire..........................61

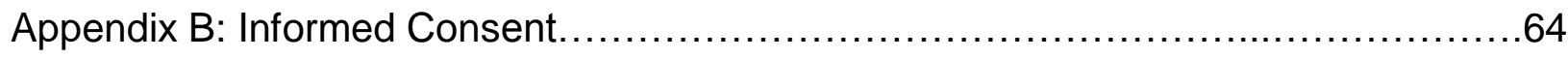

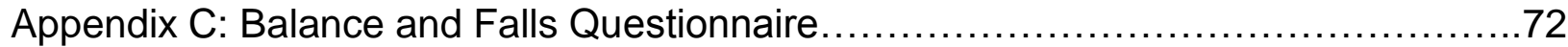

Appendix D: Urinary Incontinence Questionnaire ...................................74

Appendix E: Post-Testing Urinary Incontinence Questionnaire........................77

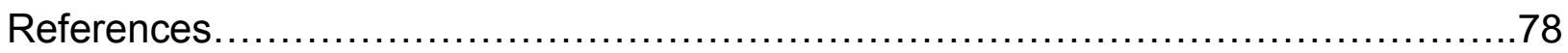




\section{Chapter 1: Introduction}

Pelvic floor dysfunction (PFD) is a general term that encompasses a plethora of physical disturbances including, but not limited to, urinary incontinence, fecal incontinence, pelvic organ prolapse, and high tone dysfunction (1). PFD is often attributed to a weakness/dysfunction of the pelvic floor muscles, specifically the pubococcygeus and iliococcygeus that make up the levator ani, coccygeus, and the surrounding connective tissue (1).

Based on previous U.S. Census Bureau data in conjunction with estimates of the incidence of various diseases and disorders from the National Health and Nutrition Examination Survey, the prevalence of PFD is on the rise. It has been predicted that by the year 2050, 43.8 million women 20 years and older nationwide will be affected by and show symptoms of various types of PFD (2). This is a vast increase over the 28.1 million women estimated to have PFD in 2010 (2).

The incidence of PFD has been shown to increase with age (3). In fact, in 2008, $40 \%$ of women in the United States ages 60 to 79 and nearly $50 \%$ of women 80 years and older demonstrated PFD (1). Although most prevalent in older populations of women, PFD is not exclusively linked to age, but other factors such as pregnancy and childbirth (3).

Regardless of age, various symptoms mark PFD. Urinary incontinence (UI) is most often studied and reported as a diagnostic factor. Between the years of 2010 and 2050, a 55\% increase in the prevalence of $\mathrm{UI}$ in American women 20 years and older has been projected (2). PFD is not gender specific, however, it has been reported that in terms of $\mathrm{UI}$, women are more frequently affected (3). In addition to age and gender, 
other potential risk factors of UI include gender, childbirth, menopause, obesity, functional impairment, and genetics (4). Athletes required to run, jump, and perform impact landings, particularly gymnasts, basketball players, and track runners have also been reported to experience UI (5).

Bladder leakage may not be the only health consequence associated with UI. Compromised postural stability in patients with PFD has been a clinical observation by one of the authors (KT) of this study. Additionally, as noted by Chiarelli et al., women with certain types of UI, specifically urge incontinence, have insufficient postural stability, as evidenced by the incidence of falls (6). Due to their anatomical location, the muscles that comprise the pelvic floor may play a vital role in the way in which stability is maintained throughout the course of everyday activities. The pelvic floor muscles contribute to the modulation of intra-abdominal pressure, which has been noted to help control the spine (7). Further distinguishing the postural functions of the pelvic floor muscles is their co-contraction with the transverse abdominis and internal obliques (8). So, in the current study, in order to see if the dysfunction/weakness of the pelvic floor muscles in women with UI compromises their hip strength and core stability, postural stability was evaluated both statically and dynamically.

This study proposed to research the postural stability responses of women who have pelvic floor dysfunction, specifically marked stress incontinence, urge incontinence or mixed incontinence. In an effort to expand the knowledge of various symptoms of PFD, and thereby the related physical limitations, this relationship was explored. The critical need for this research is that a correlation between urinary incontinence and postural instability has the capability of being further extended to the idea that treating 
urinary incontinence may help reduce this instability and therefore decrease the risk of falling.

\section{Specific Aims}

The overall Specific Aim of this study was to examine if women with PFD, specifically as it relates to urinary incontinence, demonstrate poorer postural stability as compared to an age and parity matched control group.

\section{Specific Aim 1: Static Postural Stability}

To examine if women with urinary incontinence demonstrate poorer postural stability during a static task (i.e. quiet standing) during two visual conditions (eyes opened and eyes closed) as compared to an age and parity matched control group. Specifically, the variables of anterioposterior sway, mediolateral sway, length of the path of the center of pressure, sway velocity in quiet stance, and the area of an ellipse that encompasses $95 \%$ of the center of pressure points, known as elliptical sway area, were examined during both the eyes opened and eyes closed tasks.

Hypothesis 1: Women with urinary incontinence will exhibit poorer postural stability during a static task in both the eyes opened and eyes closed conditions as compared to an age and parity matched control group.

Hypothesis 1.a: Anterioposterior sway will be greater in the women with urinary incontinence than in the control participants during both the eyes opened and eyes closed tasks.

Hypothesis 1.b: Mediolateral sway will be greater in the women with urinary incontinence than in the control participants during both the eyes opened and eyes closed tasks. 
Hypothesis 1.c: Length of the path of the center of pressure will be greater in the women with urinary incontinence than in the control participants during both the eyes opened and eyes closed tasks.

Hypothesis 1.d: Sway velocity in quiet stance will be greater in the women with urinary incontinence than in the control participants during both the eyes opened and eyes closed tasks.

Hypothesis 1.e: Elliptical sway area will be greater in the women with urinary incontinence than in the control participants during both the eyes opened and eyes closed tasks.

\section{Specific Aim 2: Dynamic Postural Stability}

To examine if women with urinary incontinence demonstrate poorer postural stability in response to dynamic postural perturbations (i.e. toes up and toes down tilt of the support surface) as compared to an age and parity matched control group. Specifically, the variables of initial sway, total sway, and sway velocity in a dynamic perturbation were assessed in response to both toes up and toes down perturbations. Hypothesis 2: Women with urinary incontinence will exhibit poorer postural stability in response to dynamic postural perturbations as compared to an age and parity matched control group.

Hypothesis 2.a: Initial sway will be greater following the perturbations in the women with urinary incontinence than in the control participants. Hypothesis 2.b: Total sway will be greater following the perturbations in the women with urinary incontinence than in the control participants. 
Hypothesis 2.c: Sway velocity in a dynamic perturbation will be greater following the perturbations in the women with urinary incontinence than in the control participants.

\section{Background and Significance}

\section{Postural Stability and Balance}

Postural stability and balance are two terms that are essentially synonymous with one another (9). Therefore, throughout the course of writing this proposal, the term "balance" found in any of the cited references was equated to "postural stability". Postural stability has been defined as the dynamic response to perturbations (10). Furthermore, postural stability can be a static measure, evidenced by "steadiness" (11) or the maintenance of the center of mass over the base of support (9). It is suspected that when postural stability is compromised, such as the result of a perturbation, the muscles of the pelvic floor are challenged and their activities increase (7). If a woman is incontinent, the pelvic floor muscles may be weak and/or function improperly (1) and an increased incidence of falls has been reported particularly in older adults with certain types of UI (6). An increase in falls may imply postural stability-related deficiencies.

Measurements of postural stability range from simple to complex assessments. Simple tests that indicate postural stability discrepancies include single-leg balance tests, whereas more technically complex tests are those that collect center of pressure (COP) data.

\section{Urinary Incontinence}

There are several types of UI that are characterized based on factors such as the activities that potentiate bladder leakage, the frequency of the symptoms, the amount of 
urine leaked, timing of the leaks (i.e. morning/night), and the causation (12). Various types of incontinence may be linked to the role of the pelvic floor muscles in bladder control. Due to their origins and insertions, these skeletal muscles are important in situations involving changes in intra-abdominal pressure (13). In instances when intraabdominal pressure rises (e.g. laughing, sneezing, coughing (14)), more stress is placed on the bladder sphincters; in order to maintain adequate urethral closure pressure, the pelvic floor muscles are further activated to retain proper positioning of the neck of the bladder (15), and keep the urethra closed (16). When the pelvic floor muscles demonstrate a weakness/dysfunction, they may not adequately compensate for the increased intra-abdominal pressure and UI may result.

Women with stress incontinence have increased, but delayed, pelvic floor muscle activity $(7,17)$ and increased external oblique activity associated with postural perturbations when compared to continent women (7). Therefore, it is suspected that women with PFD, especially those who are incontinent, have different postural responses to perturbations compared to women without PFD (i.e. without incontinence). Urologists and other medical practitioners can diagnose UI and prescribe further treatment. Typical treatment strategies include: 1. medication to control various causes of incontinence (e.g. anticholinergics to relax an overactive bladder (12)), 2. pelvic floor muscle training (PFMT), and 3. surgery. As $51 \%$ of women perform pelvic floor muscle contractions incorrectly (18), biofeedback techniques utilizing real-time surface EMG to visualize pelvic floor muscle recruitment are used during PFMT to enhance the individual's ability to contract and relax the target muscles (19). Currently, the purpose 
of this therapy is intended for incontinence cessation and not necessarily to correct any inconsistencies in postural stability.

\section{Significance}

To the investigator's knowledge, the effects of PFD on postural stability have not been widely researched. Observations that PFD contributes to negative symptoms such as postural instability necessitate the significance of attempting to establish this relationship. Postural instability is likely to be very limiting to a woman's everyday life activities. Activities of daily living may be compromised to such an extent as to make women unable to perform them on their own or perform them in a limited capacity.

Pelvic floor dysfunctions in 2008 affected nearly $25 \%$ of women in the U.S. (1). This number is predicted to increase rapidly. A rising number of women possessing various risk factors (2), as well as more women reporting their incontinence, may be reasons for this suspected increase. This increase could also potentially correlate with more women experiencing limited functional abilities that could possibly stem in part from weakness/dysfunction of the pelvic floor muscles. Findings from this thesis that support this correlation will offer preliminary data related to the treatment of women with postural-related issues due to their PFD. Since PFMT is currently prescribed for incontinence treatment, future studies may elucidate findings that this type of therapy may also be beneficial in reversing, or even preventing, postural instability.

Furthermore, the critical need for this research is that the findings can potentially be further extended to the idea that treating urinary incontinence may help attenuate postural instability and essentially reduce fall risk. 


\section{Assumptions}

It was assumed that all subjects were truthful in their responses about their current health and health history when undergoing the phone screening. Various assumptions were made regarding the lack of continence displayed by the subjects. More specifically, bladder leakage was not directly measured, but assumed and implied based on the subjects' prescriptions for physical therapy/PFMT and questionnaires about urinary leaks both preceding and following data collection. Subjective data about falls experienced and any instability noticed on a day-to-day basis were also collected via questionnaire; honesty in answering the questions was assumed for all participants. Experimental subjects were paired to control subjects based on parity and age; we assumed that all subjects were honest in their report of the number of children to whom they have given birth. Truthfulness in answering questions related to urination, menstruation, menopause, and the means of delivery for each child was assumed as well.

We assumed that the subjects did not participate in any physical activity prior to the postural stability testing as it could have potentially affected the results. Also, adherence to the protocol during the postural stability testing, particularly in the conditions of the static test in which the eyes were closed, had to be assumed.

\section{Limitations}

The subjects who participated in this study represent only a small portion of the women affected by and seeking physical therapeutic treatment of stress incontinence, urge incontinence or mixed incontinence. The sample size was comprised of patient referrals for treatment of these particular types of UI to one of the authors (KT) of the 
study who is a board certified specialist in women's healthcare. Therefore, the ability to extrapolate the results of this study is hindered in that it is limited to a small percentage of patients. The age distribution of the subjects recruited affects to generalization of the female population. Due to the inverse relationship between age and postural stability (20), age is a confounding factor. For instance, data from subjects 65 years or older may not be applicable to those with PFD in their teens. Similarly, body mass index (BMI) has been noted to adversely affect postural stability (20). Recruiting control subjects to match experimental subjects based on age, parity, and BMI would have been problematic and therefore this factor was not considered when seeking subjects. The results of this study are not able to be extended to the male population because all subjects were female. Lastly, the perturbations induced throughout the dynamic testing were not reflective of real-life activities that may challenge postural stability.

\section{Delimitations}

This study only included generally healthy women, ages 18 years and older, affected by stress incontinence, urge incontinence or mixed incontinence. The inclusion of generally healthy subjects was an effort to minimize any confounding health factors, other than UI, that may alter postural stability. Therefore, any findings from this study are applicable to those women who are also generally healthy, experience stress incontinence, urge incontinence or mixed incontinence, and whose age group is represented by the participants.

\section{Key Terms}

Anterioposterior sway: the range of the movement of the COP along the y-axis (i.e. foreaft) during quiet stance 
Balance (postural stability): the ability to maintain the position of the body's center of mass over the base of support (9); can be measured statically and dynamically

Center of pressure: a point of pressure exerted by the feet that can be measured using force plates; a measure of postural stability that can be thought of as the center of force Initial sway: the difference between the point at which the perturbation begins and maximum perturbation Mediolateral sway: the range of the movement of the COP along the $x$-axis (i.e. side to side) during quiet stance

Parity: the number of children to whom a woman has given birth

Pelvic floor: the muscles, specifically the coccygeus and levator ani, and the connective tissue located near the pelvis that function to support the pelvic organs and help maintain continence

Pelvic floor dysfunction: a variety of conditions/symptoms reflective of possible weakness, tightness and/or improper functioning of the pelvic floor muscles and the surrounding connective tissue

Perturbation: a disturbance of bodily equilibrium

Postural stability (balance): the ability to maintain the position of the body's center of mass over the base of support (9); can be measured statically and dynamically Sway velocity: the average velocity (in terms of displacement and time) of the COP movement; can be assessed statically and dynamically Total sway: the entire movement of the COP, marked by the difference between maximum anterior and maximum posterior COP displacement, in response to a dynamic perturbation 
Urinary incontinence: involuntary loss of bladder control accompanied by urine leakage 


\section{Chapter 2: Literature Review}

\section{Introduction}

PFD is reflective of possible weakness, tightness and/or improper functioning of the pelvic floor muscles and the surrounding connective tissue (1). Therefore, PFD is an all-encompassing term that generalizes a wide variety of health abnormalities and challenges faced by more and more women everyday.

Although the PFD symptom of UI is widely studied, it is complicated by its numerous types. Providing even further complication is the idea that UI may lead to impaired postural stability (6). The various risk factors associated with compromised postural stability have been well studied. These factors include, but are not limited to, age (22), body weight (21), gait problems (23), and muscular weakness (23). However, the idea that UI /pelvic floor muscle weakness may be another risk factor of poor postural stability adds yet another tier of complexity to this area of study and warrants further research. Throughout this literature review, any study referenced that assessed balance was noted to have assessed postural stability, as the two terms are synonymous (9).

In order to determine if a postural stability deficiency is present, basic measurement techniques are commonly used in a clinical setting. Furthermore, more complex assessments of stability involve the acquisition of COP data through the use of force plates. Both COP data obtained from static and dynamic measures can be further analyzed to gauge if stability is compromised. Tests able to acquire such data use computerized posturography, which is known for its ability to thoroughly examine stability (9). Examples of computerized posturography include the tests performed in 
the current research: the Sensory Organization Test (SOT) and the Adaptation Test (ADT).

\section{Pelvic Floor Anatomy}

\section{Musculature}

The majority of the pelvic floor is comprised of skeletal muscles including the levator ani and coccygeus. These muscles attribute to the funnel-shape of the pelvic floor (24). The levator ani is a prominent muscle of the pelvic floor that contains three different regions: iliococcygeal region, pubococcygeus muscle, and puborectalis (24, 25). In females, it surrounds the vagina, urethra, and anorectal junction (24). The origin of the levator ani is the inside of the pelvis from pubis to ischial spine, whereas its insertions are the inner surface of coccyx, the obturator internus, the levator ani of the opposite side of the pelvic floor, and the structures that penetrate it (24). The obturator internus acts as a bridge between the pelvic floor and hip (24). The coccygeus has a pronounced triangular shape that is located posteriorly to the levator ani (24). The coccygeus originates at the spine of the ischium and inserts into the sacrum and coccyx (24).

\section{Function}

The levator ani and coccygeus perform several actions simultaneously. Hodges et. al showed that the pelvic floor muscles may contribute to the activity of the trunk in circumstances when stability is challenged (13). The pelvic floor muscles co-contract with the transverse abdominis and internal obliques (8) to contribute to the maintenance of stability. Together these muscles also help maintain the structure of the pelvic floor, support the pelvic viscera (24), and assist in proper positioning of the bladder neck, particularly when intra-abdominal pressure rises (15). However, in addition to working 
together, they also perform actions relative to their locations, origins, and insertions. Specifically, the levator ani functions to help keep the urethra closed, especially when intra-abdominal pressure is increased (16); it also forms sphincters located at the anorectal junction and vagina, and lifts the anal canal during defecation (24). The coccygeus is important in supporting the coccyx and pulls it forward in response to events that cause it to shift posteriorly, such as defecation and childbirth (24).

\section{Specific Pelvic Floor Dysfunctions}

\section{General Information}

Urinary incontinence, fecal incontinence, and female pelvic organ prolapse are three main symptoms of pelvic floor dysfunction (1). PFD has a high prevalence in females (3), and in 2008 affected approximately one quarter of women in the U.S. (1). This increasing problem has been found to contribute to a decrease in the quality of life (3).

\section{Risk Factors}

MacLennan et al. found that factors such as gender, aging, and pregnancy are among the characteristics that increase the risk of PFD (3). With pregnancy and childbirth, all modes of delivery tend to increase the likelihood of obtaining a PFD (3). In fact, when compared to nulliparous women, women who had given birth either via caesarean section, spontaneous vaginal delivery, or instrumental delivery had significantly higher associations with PFD (3). This may be attributed to the hormone relaxin as its production is generally increased during pregnancy (26). Relaxin has also been shown to act on the pelvic floor's connective tissue, potentially making this hormone, in part, responsible for the relationship between pregnancy and PFD (26). 
However, it has also been shown that UI due to childbirth often subsides puerperium (i.e. about 6 weeks post-partum) (27).

\section{Fecal Incontinence}

An estimated 18 million adults reported fecal incontinence in 2009 (28). The National Digestive Diseases Information Clearinghouse has extensively outlined fecal incontinence and identified it as the incapability to control or hold a bowel movement (29). Demonstrating such disparities in rectal functioning can cause a decrease in one's quality of life (30).

Fecal incontinence can also directly result from damage or weakness of the pelvic floor muscles (29). Malfunctions of the pelvic floor may be responsible for the body's impaired ability to recognize that stool is present and the formation of decreased or weaker muscle contractions during defecation (29).

Aside from pelvic floor-related issues, there are other health problems that seem to cause fecal incontinence. Some of these problems are diarrhea, nervous system injury, and an overall poor health status that includes multiple chronic diseases (29).

Additionally, age is directly correlated with an increasing risk of this particular type of incontinence (28), as is being female (29).

Treatment of fecal incontinence depends on the underlying cause(s); it can be as simple as modifying diet or as extensive as surgery (29). Other possible forms of treatment are medications, exercises to strengthen and retrain the pelvic floor, and electrical stimulation (29).

\section{Female Pelvic Organ Prolapse}

The female pelvic organs include the uterus, bladder, rectum, and vagina $(1,24)$. 
The pelvic floor muscles along with the corresponding ligaments and fascia help keep these organs in the proper place (25). But, if the pelvic floor muscles are not properly functioning (e.g. they are weakened), they lose their ability to maintain the appropriate positioning of the organs, which eventually stretches the connective tissue and leads to their prolapse, or descent (25), causing protrusion of the organs into the vaginal canal and at times subsequent discomfort (1). Those with pelvic organ prolapse may have developed elimination dysfunctions, reported difficultly participating in physical activity, and may have experienced restricted sexual functioning (1).

Vaginal deliveries have been reported to increase the risk of obtaining pelvic organ prolapse, which becomes especially evident later in life (25). Kim et al. reported that age, parity, and menopause are also correlated to female pelvic organ prolapse (31).

There are a few common detection methods for pelvic organ prolapse. Physical examinations can be conducted, such as the Pelvic Organ Prolapse System (32). In order to more accurately gauge the severity of the disorder, a technique called dynamic cystoproctography can be conducted $(33,34)$. Dynamic cystoproctography is essentially an imaging technique of specific areas of the pelvic floor used to quantify and provide a visual representation of the prolapse (33). Once detected and quantified, surgical procedures may be recommended. Olsen et al. looked at the epidemiology of surgical treatment of pelvic organ prolapse and reported that the need for multiple operations is not uncommon in an attempt to correct this specific PFD (35).

\section{Urinary Incontinence}

The third main symptom of PFD is urinary incontinence. This disorder seems to be frequently studied and was the focus of this research, as it served as inclusion 
criteria for subject participation in the study.

The term UI does not specifically refer to only one type of incontinence. In fact, according to the National Kidney and Urological Diseases Information Clearinghouse, seven variations of this dysfunction exist and are categorized based on a variety of characteristics (12). Stress incontinence is distinguished by a small amount of urinary leakage during various movements, particularly coughing, sneezing or physical activity (12). Urge incontinence is characterized by the presence of a strong urge to urinate (12). Overactive bladder involves frequent urination that may be present in conjunction with urge incontinence (12). Functional incontinence describes the inability to toilet at the appropriate time due to physical limitations, disabilities, impaired thought or communication, or obstacles outside of one's control (12). Mixed incontinence is the combination of stress and urge incontinence (12). Overflow incontinence is the product of a full bladder in which some excess urine is leaked. Finally, transient incontinence is both temporary and dependent on certain situations (e.g. infections or newly prescribed medications) (12). Although UI includes several classifications, Handa et al., whose conclusions did not distinguish between these various types, reported a decreased quality of life in women with UI (30). The current study only recruited women with stress incontinence, urge incontinence or mixed incontinence.

Women, more so than men, are affected by UI (3) and it may be partly attributed to each genders different urinary tract anatomies (12). Along with gender, there are many other risk factors; some of them include age, pregnancy, hormonal changes, obesity, functional impairment, and genetics $(4,26)$. 
Evidenced via magnetic resonance imaging, it has been shown that there is a link between leukoaraiosis (i.e. changes in cerebral white matter) and urge incontinence in older individuals (mean age of 73 years old) (36). This particular correlation suggests that there is a possible interaction between the central nervous system, particularly the brain, and the symptoms of incontinence (36). Information related to the brain was not a variable in the current study, but it is important to note its potential connection to UI.

Although most of the focus of UI studies seems to be on the aging population, there are data reporting that younger female athletes have also been affected (5). Athletes participating in higher impact athletics may leak urine with running, jumping, and landing (5). It is suspected that high impact activities contribute to pelvic floor muscle weakening.

Diagnostic measures of $\mathrm{UI}$ are generally initiated once the patient has noticed symptoms of urine leakage. Physicians will often begin by having patients complete a bladder diary, tracking the times and amounts of urinary release over several days (12). There are also different tests that can be conducted to better indicate the type of incontinence that is being experienced. For instance, a bladder stress test may be performed requiring forced coughing in conjunction with monitoring volume of urine loss (12). Other methods such as ultrasounds of the urinary tract, cystoscopy of the urethra and bladder, and urine cultures are used (12).

Treatment is possible for all kinds of $\mathrm{UI}$ and can be done at any age (12). Medications for the different types, such as anticholinergics for an overactive bladder to decrease bladder spasms, can be prescribed (12). Pelvic floor muscle exercises, such as muscular contractions, can be done on a daily basis in an effort to strengthen the 
weakened pelvic floor muscles (12). However since many women perform these contractions incorrectly (18), biofeedback, utilizing an internal sensor, is a useful modality to teach the patient to correctly activate the muscles to alleviate incontinence; this feedback has been shown to have more effectiveness compared to other forms of treatment including pharmacological interventions, electrical stimulation, and surgery (19).

\section{Urinary Incontinence and Muscle Activity}

Continent women tend to have increased pelvic floor muscle activity upon postural tasks aimed at challenging stability, such as repetitive arm movements (13). Since some incontinent women demonstrate weakness/dysfunction of the pelvic floor muscles (1), it would be logical to hypothesize that muscle activity decreases upon perturbations or tasks that compromise stability. However, contrary to this hypothesis, Smith et al. found that the activity of the pelvic floor muscles in women experiencing stress incontinence increases even more than that of continent women in response to postural perturbations $(7,17)$. It has also been shown that there is delayed activity of the pelvic floor muscles upon perturbation (i.e. rapid arm movements) in women with stress urinary incontinence (17).

A possible explanation for the differences in activity magnitude when comparing incontinent to continent subjects is that when the activity of the pelvic floor muscles increases, certain abdominal muscle activity, particularly in the external obliques, simultaneously gets larger $(7)$. These increased activities $(7,17)$ further cause intraabdominal pressure to rise (37) placing an even greater compensatory challenge on the pelvic floor muscles thereby possibly provoking incontinence (7). During a perturbation, 
the responsiveness and activity onset of the pelvic floor muscles may be crucial in the maintenance of continence and lumbopelvic stability (17). In other words, if the response is delayed, possible effects are incontinence as well as instability (17).

It has also been found that when the severity of incontinence is taken into account, as severity increases, the activity of the external oblique muscle (7) and essentially intra-abdominal pressure increase even more (37). Therefore, women with severe incontinence may have pelvic floor muscles that, despite increased activity, are less effective at compensating for the other increases (i.e. external oblique activity and intra-abdominal pressure) (7).

\section{Urinary Incontinence and Postural Stability}

As previously outlined, $\mathrm{UI}$ and its various types demonstrate a wide range of symptoms related to specific factors. However aside from the symptom of actual urine excretion, UI may be related to other negative consequences. Particularly, postural stability, under investigation in the current study, may be compromised in women lacking continence (6).

As mentioned, delayed onset of pelvic floor muscle activity might lead to lumbopelvic instability (17). But in addition to assessing muscle activity onset, evaluating the incidence of falling may provide further insight into various postural stability issues. Lack of stability is associated with an increased incidence of falls (38). The finding that aging adults experience an increased number falls resulting from compromised equilibrium (i.e. instability) furthers support this relationship (22). In other words, this particular population is commonly affected by stability-related deficiencies evidenced by falling (22). Similarly, in an assessment of a number of studies, 
Rubenstein cited a comprehensive table of risk factors for falling; impaired balance (i.e. instability) was included (23).

Urinary incontinence was not found on the table of fall risk factors specifically mentioned above (23). But, Chiarelli et al. chose to review the related literature in order to find some sort of correlation between these two variables (6). Upon review, it was found that there seems to be an association between incontinence, particularly urge incontinence, and falling (6). However, this review did not include a wide age range, therefore this may have been a limitation of the study (6). If the literature that was reviewed included more than just "older adults", although the specifications of "older" were not defined, there may have been more of a trend toward a correlation between falling and the particular forms of UI that were evaluated. Therefore, the current study included a more diverse demographic by broadening the age range.

\section{Postural Stability Measurement Techniques}

The assessment of postural stability can be done though a variety of modalities. Clinical assessments tend to be simplistic in administration as well as based more on observation rather than quantitative data. To explore postural stability with further complexity, technical procedures including static and dynamic assessments can help detect deficiencies. Sometimes certain analyses will incorporate the assessment of postural control, which focuses more on integrated processes (e.g. motor control strategies) that contribute to stability (9). However, these processes were not directly examined in the current research. 


\section{Clinical Assessments}

Assessments of postural stability in a clinical setting are geared toward the risk of falls and possible determination of the causes of various balance disorders (39). One subjective way to assess stability is done via questionnaires (9). For example, the Activities-specific Balance Confidence (ABC) scale helps to identify the fear of falling in older individuals, particularly during activities of daily living $(9,40)$. Functional assessments, used clinically due to their simplicity, are another way to evaluate postural stability (9). Basically, functional assessments investigate the risk of falling and an apparent need for rehabilitation, however they do not offer a way to detect or decipher between different types of balance disorders $(39,41)$. Some examples of functional tests include: the single-leg stance duration test (39) and the Time Up and Go (TUG) Test (42). Another category of stability assessments used clinically is systems assessments that provide insight into different balance disorders to detect the appropriate causes (e.g. sensorimotor impairments) in an effort to help with treatment $(39,41)$. The Balance Evaluation Systems Test (43) and the Physiological Profile Approach (44) are examples. The last kinds of assessments are objective and can either rely on the use of inertial sensors during certain tasks or computerized testing (i.e. computerized posturography) to look at both static and dynamic measures of postural stability (39). Inertial sensors can provide additional feedback on limb and torso movement during various stability tests and help measure gait (39); they are often used clinically due to their cost effectiveness (39). Computerized posturography, specifically related to static and dynamic testing, are further explained in the following sections. 


\section{Static Postural Stability Assessment}

Measures of static postural stability are obtained from COP data acquired through the use of force plates. For clarity, static postural stability has been described as postural steadiness (10) and is generally quantified in a quiet stance (39). In a review of literature, it was found that various measures obtained from force plates seem to be effective in their prediction of falls (38). There are several variables that can be computed from COP data to provide insight into one's stability. To date, no studies in the refereed literature have quantified static postural stability using COP data as it relates to those demonstrating PFD. But several other investigations of stability on different populations have used static COP measures similar to those that were used in the current study.

Due to the equipment available, the current study utilized the SMART Balance Master (NeuroCom, Clackamus, OR) to perform the Sensory Organization Test (SOT) for the purpose of gathering all COP measurements on its force plates. Buatois et al. concluded that, when compared to other clinical tests, the SOT was a better measure of predicting the incidence of falling in subjects over the age of 65 (45). The SOT requires the subjects to undergo various challenges to their postural stability through the use of six different conditions. The six conditions are as follows: 1 . Eyes open, visual surround fixed, surface fixed, 2. Eyes closed, visual surround fixed, surface fixed, 3. Eyes open, visual surround moving, surface fixed, 4. Eyes open, visual surround fixed, surface moving, 5. Eyes closed, visual surround fixed, surface moving, and 6. Eyes open, visual surround moving, surface moving. More information than is relevant to the current study was gathered from these six conditions, however the static COP data necessary 
for this research were obtained from a quiet stance with eyes opened (i.e. condition 1) and eyes closed (i.e. condition 2).

Postural sway is an example of one static variable and is measured by finding the displacement of each COP point throughout a quiet stance (39). Postural sway can be further dissected into anterioposterior and mediolateral components by assessing the COP movement along the $y$-axis and $x$-axis, respectively. The use of mediolateral COP measures has been highlighted as an effective measurement to forecast falls (38) and essentially assess stability. Additionally, Horak et al. measured anterioposterior sway during eyes open and eyes closed conditions on a stable surface (46). In this same study, COP velocity was measured and anterioposterior sway for these two visual conditions was used to better quantify COP displacement (46); these two variables, comparable to sway velocity in a quiet stance and length of the path of COP, respectively, were both measured in the current study. To assess postural stability after various exercise protocols, one study used the statistical analysis of elliptical sway area of $95 \%$ of the COP data (47). Calculations that utilize ellipses can further evaluate postural sway in terms of its direction and size, as well as provide a multidirectional analysis (48).

Lord and Menz reported that as a person ages, visual inputs may often weaken, resulting in subsequent declines in postural stability (49). In another study that compared the postural control of older adults without vision impairments to older adults with visual impairments (i.e. low vision and blindness), it was reported that the subjects with visual impairments had greater body sway (50). Ultimately, it was concluded that older adults with low vision or blindness have decreased postural control when 
compared to older adults with unimpaired vision (50). Although these two studies focused on an aging population and their biological visual disparities $(49,50)$, this idea can likely be extended to any age group with any sort of visual impairment, whether controllable (e.g. closing the eyes) or uncontrollable (e.g. a physical limitation).

Therefore, in the current study, the use of eyes open and eyes closed conditions added a complex visual dimension to each static variable.

Based on the works of other investigators, it can be rightfully concluded that anterioposterior sway, mediolateral sway, length of the path of COP, sway velocity in quiet stance, and elliptical sway area are all valid variables that can be used for the evaluation of static postural stability in both eyes closed and opens conditions.

\section{Dynamic Postural Stability Assessment}

Even though static assessments offer necessary variables to quantify postural stability, it is also important to include dynamic assessments in order to draw more substantiated conclusions. The dynamic assessment of COP responses to perturbations provided a more extensive look at stability. To the author's knowledge, the Adaptation Test (ADT), which was performed in the current study on the SMART Balance Master, has not been cited as a method for measuring postural stability specifically in women with PFD. However, the same variables that were obtained from the ADT have been used to explore postural stability in other studies about various populations.

Nakamura et al. used measurements from perturbations of force plates in order to examine sway in healthy young subjects versus healthy older subjects; they determined that sway was a valid means of measuring postural control (51). In both 
groups, the perturbation of the support surface tended to increase COP displacement (51). In terms of sway, the subjects in the older adult group experienced more of an increase in sway compared to the younger group (51). These findings correlate to the idea that age and postural stability are inversely related (22), but add a dynamic component that shows that individuals at risk of falling (i.e. older adults) have different responses to perturbations (51). Although in an assessment of pregnant women, McCrory et al. used COP data from responses to perturbations to study initial sway, sway velocity, and total sway (52) - all of the dynamic variables in the current study. It was reported that pregnant women who have fallen have different postural stability responses to perturbations compared to pregnant non-fallers, as evidenced by the dynamic variables noted above (52).

The fact that the ADT delivers some unexpected perturbations of the force plate is an important part of a dynamic postural stability assessment. Actually, it has been previously reported that both incontinent and continent women showed a greater amount of pelvic floor activity when the postural challenge was unexpected versus when it was expected (7). Therefore, in our study, the unexpected perturbations delivered throughout the ADT helped to better simulate a true postural response and not one that was anticipated by the subjects. 


\section{Chapter 3: Research Methods and Procedures}

\section{General Research Outline}

The primary research objective of this study was to determine if women with PFD, specifically as it relates to stress incontinence, urge incontinence, and mixed incontinence, have deficits in postural stability during static tasks and in response to postural perturbations of the support surface. There is a critical need for this research in that a correlation between urinary incontinence and postural instability has the capability of being further extended to the idea that treating urinary incontinence may help attenuate postural instability, thereby reducing fall risk.

Seven women with stress incontinence or mixed incontinence were recruited from the clinical practice of one of the primary investigators (KT); no subjects had an urge incontinence diagnosis. An age and parity matched control group was recruited by word of mouth from the greater Morgantown area. Data collection took place in the Balance and Falls Laboratory at West Virginia University. A SMART Balance Master (see Figure 1) was used to assess postural stability via static and dynamic variables calculated from COP data. COP movement was assessed during a quiet stance in eyes open and eyes closed conditions. COP movement in response to toes-up and toesdown tilt of the support surface was measured. All subjects completed questionnaires related to health history, exercise, balance and falls, incontinence, menstrual history, and pregnancy history. A two-factor MANOVA (group, eyes open/closed) was performed on the following static measures of postural stability: anterioposterior sway, mediolateral sway, length of path of COP, sway velocity in quiet stance, and elliptical sway area. A second two-factor MANOVA (group, toes-up/down tilt) was performed on 
the following measures of dynamic postural stability in response to the perturbation: initial sway, total sway, and sway velocity in a dynamic perturbation. The alpha value for the statistical analyses was set at 0.05 .

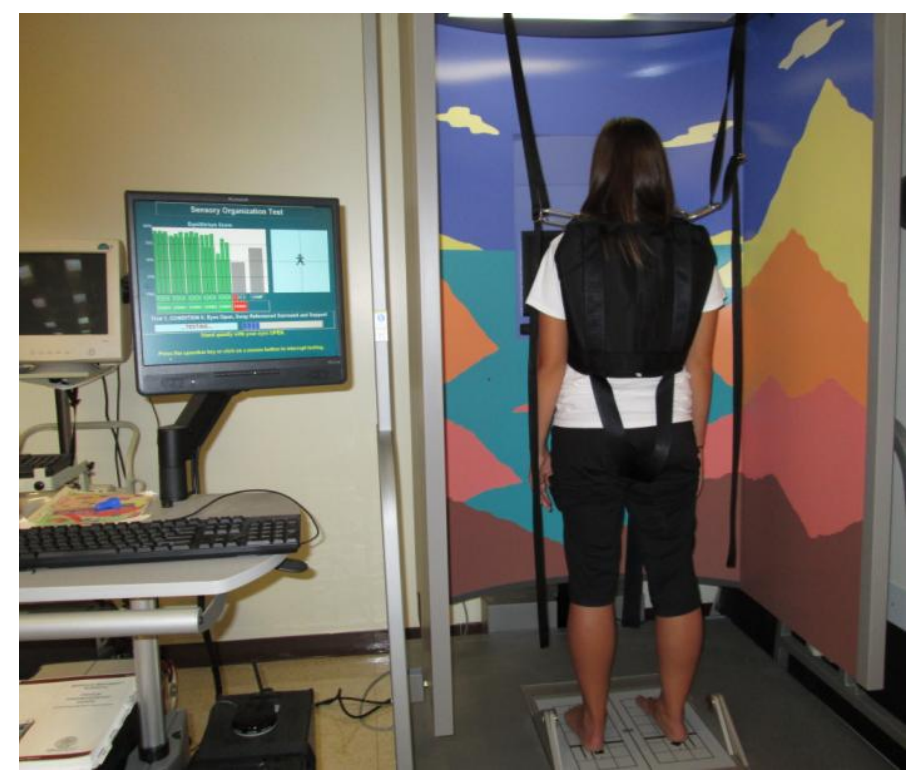

Figure 1: NeuroCom's SMART Balance Master in the Balance and Falls Laboratory at West Virginia University

\section{Subjects}

Seven women 18 years and older comprised the experimental group of this study. The experimental subjects only came from women who were referred for incontinence treatment to one of the primary investigators (KT) at West Virginia University Cheat Lake Physicians. It is important to note that only women with certain types of urinary incontinence (i.e. stress incontinence, urge incontinence, and mixed incontinence) were included. An age and parity matched control group was also recruited by word of mouth from West Virginia University and the greater Morgantown area. All participants were evaluated using the following specific inclusion and exclusion criteria. 


\section{Subject Inclusion/Exclusion Criteria}

The participants of the experimental group of this study met all of the following criteria:

1. Female

Rationale: Only women who had a medical diagnosis of UI were included in this study since this gender is most commonly affected by PFD (3).

2. At least 18 years old

Rationale: The age range was broad considering that incontinence can affect a wide range of women. It was important to include an older population since age and menopause are both risk factors of $\mathrm{UI}(4)$.

3. Clinical diagnosis of stress incontinence, urge incontinence or mixed incontinence

Rationale: Only women affected by stress incontinence, urge incontinence or mixed incontinence were included in the experimental group since it has been clinically observed, as well as shown by Chiarelli et al., that these particular forms of PFD may attribute to postural issues (6).

4. Any means of delivery by which the women have borne children Rationale: According to MacLennan et al., the prevalence of PFD increases in women who have been pregnant, despite the mode of delivery (3). Therefore, it was not necessary to exclude, or pair subjects, based on this factor.

Women were excluded from this study if they met one or more of the following criteria:

1. Women who were currently pregnant 
Rationale: Pregnant women tend to have alterations in various measures of postural stability (53), therefore their postural responses would have been likely influenced by additional factors outside of UI.

2. Women who were 3 months or earlier post-partum

Rationale: It has been shown that pregnancy is correlated to $\mathrm{UI}$, but it often subsides puerperium (i.e. about 6 weeks after delivery) (27). However, in order to ensure that the current study did not include women whose incontinence was only temporary due to childbirth, we were advised by one of the primary investigators (KT) to exclude women who were 3 months or less post-partum.

3. Past hysterectomy procedure

Rationale: Women who have had a hysterectomy were excluded in order to alleviate an inability to find a control subject to match age, parity, and a hysterectomy procedure.

4. Balance disorders, persistent dizziness, vertigo, neuromuscular impairment or neurological disorder

Rationale: Such health problems could have led to postural responses independent of the pelvic floor muscles and therefore unrelated to UI.

5. History of uncontrolled high blood pressure, diabetes (and subsequent use of insulin), heart attack, bypass, angioplasty or heart problems (e.g. congestive heart failure, angina, irregular heart beat)

Rationale: Potential subjects with these conditions had the potential to need additional medical attention throughout the course of the postural stability testing. 
6. Back surgery within the past year, musculoskeletal dysfunctions or lower extremity injuries that affect gait or require limited load bearing Rationale: Each subject was required to stand while completing the postural stability testing protocols; certain issues related to the lower extremities or the back could have resulted in an inability to remain standing and/or postural compensation not involving the pelvic floor muscles.

7. Inability to stand unassisted for an extended duration Rationale: The postural stability testing protocols required the subjects to stand unassisted on a platform and undergo various perturbations; if a subject would have been unable to stand without any form of assistance, they would not have been able to complete the required tasks.

\section{Subject Recruitment Strategy}

Experimental subject recruitment was done primarily through patient referrals for stress incontinence, urge incontinence, and mixed incontinence. The control subjects were recruited by word of mouth from the Morgantown community.

\section{Subject Demographics}

Table 1 details the UI and control group demographics. There were differences between groups for mass $(p=0.036)$ and $B M I(p=0.050)$; the $U I$ group had more mass and therefore higher body mass indices as well. 
Table 1: Subject Demographics

\begin{tabular}{|c|c|c|c|}
\hline & $\begin{array}{c}\text { Ul Group } \\
(\mathbf{n}=\mathbf{7})\end{array}$ & $\begin{array}{c}\text { Control Group } \\
(\mathbf{n}=\mathbf{7})\end{array}$ & $\mathbf{p}$-value \\
\hline Age (yrs) & $51.57 \pm 17.85$ & $51.71 \pm 18.20$ & 0.988 \\
\hline Height (cm) & $164.94 \pm 5.40$ & $163.29 \pm 6.64$ & 0.620 \\
\hline Mass (kg) & $79.38 \pm 19.45$ & $60.47 \pm 8.32$ & $\mathbf{0 . 0 3 6}$ \\
\hline Body Mass Index (BMI) & $28.97 \pm 6.51$ & $22.77 \pm 3.81$ & $\mathbf{0 . 0 5 0}$ \\
\hline Number of Falls & $0.714 \pm 1.89$ & $0.00 \pm 0.00$ & 0.337 \\
\hline Numbers of Pregnancies & $1.29 \pm 0.76$ & $1.29 \pm 0.76$ & 1.000 \\
\hline
\end{tabular}

\section{Experimental Procedure}

\section{General Health Phone Screening Questionnaire}

In order to ensure that each participant had no underlying health issues that may have complicated this study or confounded the results, the subjects were contacted by one investigator and asked a series of questions regarding their current health and health history. Some of the responses (e.g. those related to exercise and weight) were used for insight into various risk factors of PFD. Appendix A contains a copy of the General Health Phone Screening Questionnaire.

\section{Informed Consent}

Before the completion of any written questionnaires or testing, the subjects signed an informed consent detailing all aspects of the study. In addition to written explanations, the procedures were also verbally detailed before they were performed. Subjects were encouraged to ask any questions that arose. The Institutional Review Board of West Virginia University approved this informed consent. Appendix B contains a copy of the informed consent. 


\section{Balance and Falls Questionnaire}

The women filled out a questionnaire related to any noticeable balance discrepancies experienced on a day-to-day basis since the onset of their UI symptoms. The term "balance" was used on this questionnaire to enhance the subjects' understanding of each question since the words "postural stability" are not as commonly used in every day language. There was also a component related to the incidence of falls, and whether or not a fall(s) had been experienced since they noticed their symptoms. On this particular questionnaire, a fall was defined as a loss of balance resulting in some part of the body - other than a foot (or feet) - touching the ground. Most of the responses from this questionnaire were not statistically analyzed, but used for the purpose of gathering subjective information. However, the number of falls reported was analyzed between groups. A copy of the Balance and Falls Questionnaire can be found in Appendix C.

\section{Urinary Incontinence Questionnaire}

Participants also completed a questionnaire, adapted from Hulme (54), which provided insight about their experiences with urinary incontinence. There were also questions regarding menses, menopause, and childbirth that provided insight into potential risk factors associated with being incontinent. Some of the answers helped in pairing the experimental subjects to the control subjects. No statistical tests were performed on the responses, but the answers to each of the questions provided subjective information related to incontinence. A copy of the Urinary Incontinence Questionnaire can be found in Appendix D. 


\section{Sensory Organization Test (SOT)}

The SMART Balance Master is located in the Balance and Falls Laboratory at West Virginia University. The SMART Balance Master can be used for a variety of different testing protocols. For the purpose of obtaining data about static postural stability, in terms of anterioposterior sway, mediolateral sway, length of the path of COP, sway velocity in quiet stance, and elliptical sway area, the SOT was performed. COP data was acquired from the SMART Balance Master by the 18 " x 18 " force plates that the participants stood on throughout the duration of the testing. In addition to static measurements, there are several other variables that can be obtained from the SOT, but this study was only interested in the COP measurements from quiet stance during eyes open and eyes closed conditions. However, due to the software constraints of the SOT, the testing had to be fully completed and was unable to be stopped even after the data necessary for this study were acquired. The reliability (ICC) of clinical low back pain patients, another population with balance impairment, in condition 1 of the SOT was 0.56 and in condition 2 it was $0.77(55)$.

Each subject was required to wear a harness, provided by NeuroCom, to attenuate any falls that may have occurred during testing. After donning the harness, the subjects stepped up onto the force plate and were further secured using metal carabiners from each side of the harness to the SMART Balance Master. The feet were placed hip width apart. Also, alignments of the medial malleolus with the middle of the force plate and the lateral calcaneus with the appropriate height line were ensured. Once in the correct position, the subjects were encouraged to remain still and not deviate from their current stance. 
The SOT required the subjects to experience various situations involving six different conditions, which are listed below; the first two conditions were of primary importance for obtaining the appropriate static data in the current study.

1. Eyes open, visual surround fixed, surface fixed

2. Eyes closed, visual surround fixed, surface fixed

3. Eyes open, visual surround moving, surface fixed

4. Eyes open, visual surround fixed, surface moving

5. Eyes closed, visual surround fixed, surface moving

6. Eyes open, visual surround moving, surface moving

Due to the nature of the pre-programmed protocol, each subject underwent testing that assessed somatosensory, visual, and vestibular inputs on postural stability. As mentioned, some of the results, such as those directly related to inferences about vestibular contributions to postural stability, were not primary variables in this study, but these data may prove to be useful in future research.

The instructions for each condition were verbally explained to the participants. If they had any further questions about the test protocols, they were encouraged to ask the investigator prior to the commencement of the particular condition.

Each of the six conditions consisted of three, 20-second trials; in those that required a moving surface (conditions 4-6), there was a slight rotation of the force plate. Despite any closing of the eyes, movement of the visual surroundings or rotation of the force plates, the subjects were to try to maintain stability throughout each of the conditions. Normally, the SOT allows the participants to track their progress through the use of a 
computer screen showing their general results after each trial. However, for the purpose of this study, that screen was shut off as to not provide any feedback that may have enhanced the subjects' responses.

In order to analyze static postural stability by variables including anterioposterior sway, mediolateral sway, length of the path of COP, sway velocity in quiet stance, and elliptical sway area, raw COP data were processed from conditions 1 (static, eyes open) and 2 (static, eyes closed).

To determine anterioposterior sway, the minimum y coordinate was subtracted from the maximum y coordinate.

Anterioposterior sway $=\max _{\mathrm{y}}-\min _{\mathrm{y}}$ Similarly, to determine mediolateral sway, the minimum $x$ coordinate was subtracted from the maximum $x$ coordinate.

Mediolateral sway $=\max _{x}-\min _{x}$ The length of the path of COP was found using the COP $x$ and $y$ coordinates for each time point throughout the testing. The $x$ and $y$ coordinates reflect mediolateral sway and anterioposterior sway, respectively. The $x$ and $y$ values from time point one and time point two were used to calculate the displacement by the Pythagorean Theorem; this simple calculation continued with each consecutive time point. Once all of the displacements were calculated, their sum reflects the length of the path of COP.

Length of path of COP $=\Sigma$ displacement $t_{n}=\sqrt{ }\left(\left(x_{n}-x_{n-1}\right)^{2}+\left(y_{n}-y_{n-1}\right)^{2}\right)$

Sway velocity in quiet stance was calculated by dividing COP displacement by time. More specifically, displacement calculations needed for sway velocity were the same as those performed for the length of the path of COP. Therefore, the change in the $x$ and $y$ 
coordinates from each consecutive time point was applied to the Pythagorean Theorem to find the displacement. All of these values were then summed to achieve total displacement. The change in time was calculated by subtracting the initial time point from the final time point.

$$
\text { Sway velocity } \text { quiet stance }=\frac{\text { Displacement }}{\Delta \text { Time }}
$$

Elliptical sway area was found using a statistical analysis tool that fits an ellipse around $95 \%$ of all of the COP data points $(56,57)$; the area of that ellipse was then calculated using principal component analysis (58). Figure 2 details the components of an ellipse as they relate to COP. The purpose for conducting an elliptical sway area calculation was to examine sway in a more directional manner rather than just in terms of the $x$ and y coordinate planes; elliptical sway area gave a more dimensional measure of sway.

Area of an Ellipse $=\pi^{*}$ radius $1^{*}$ radius2

'Radius 1 ' is the radius of the long axis and 'Radius 2 ' is the radius of the short axis of the ellipse.

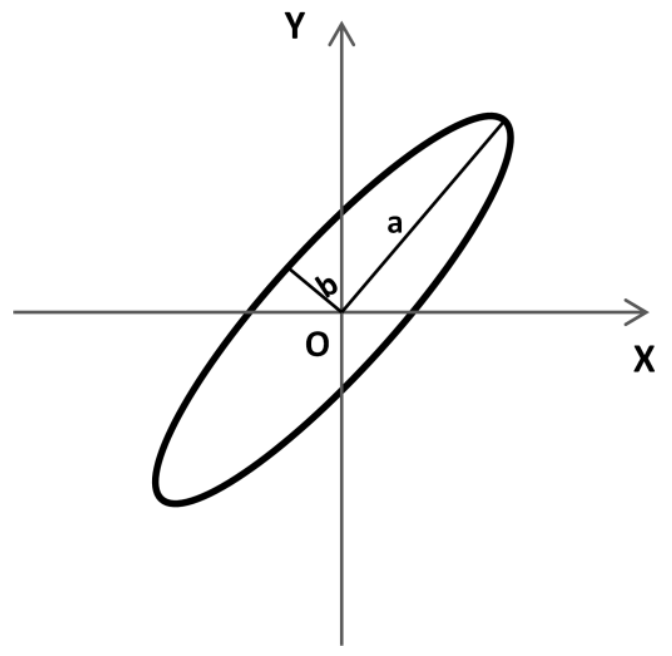

Figure 2: General ellipse diagram as it relates to COP data; $x$-axis shows mediolateral sway, $y$-axis shows anterioposterior sway, $O$ : origin at which the ellipse is centered, $a$ : length of the major axis (with respect to the origin), b: length of the minor axis (with respect to the origin) 


\section{Adaptation Test (ADT)}

In addition to the SOT, the ADT was performed on the SMART Balance Master in West Virginia University's Balance and Falls Laboratory. The ADT protocol was used to examine dynamic measures of stability including initial sway, total sway, and sway velocity in a dynamic perturbation. The starting position for the test required a hip width stance with the medial malleolus lined up with the middle of the 18 " $\times 18$ " force plate and the lateral calcaneus aligned at the appropriate line. A harness for safety and prevention of falls was worn and attached on both sides to the SMART Balance Master throughout the entire testing duration. Maintenance of the starting position during the course of the ADT was a primary goal. The reliability (ICC) of healthy individuals in the toes up perturbations of the ADT was 0.70 and for the toes down perturbations it was $0.65(55)$.

Before the test began, the participants were given verbal instructions. They were also given the opportunity to ask any questions related to the protocol. During the test, the platform rotated with a magnitude of $8^{\circ}$ in 400 milliseconds, which led to the surface rotating at $20^{\circ}$ per second, either toes up or toes down. The first five perturbations were toes up rotations followed by five toes down rotations. The ADT protocol was structured so that the first perturbations (i.e. first toes up rotation and first toes down rotation) were unexpected by the subject. For this study, the computer screen that normally provides feedback was turned off as to not impact the responses in any way.

Using the COP data collected throughout the ADT, initial sway, total sway, and sway velocity in a dynamic perturbation were determined. Figure 3 and Figure 4 graphically depict these dynamic postural response variables. 


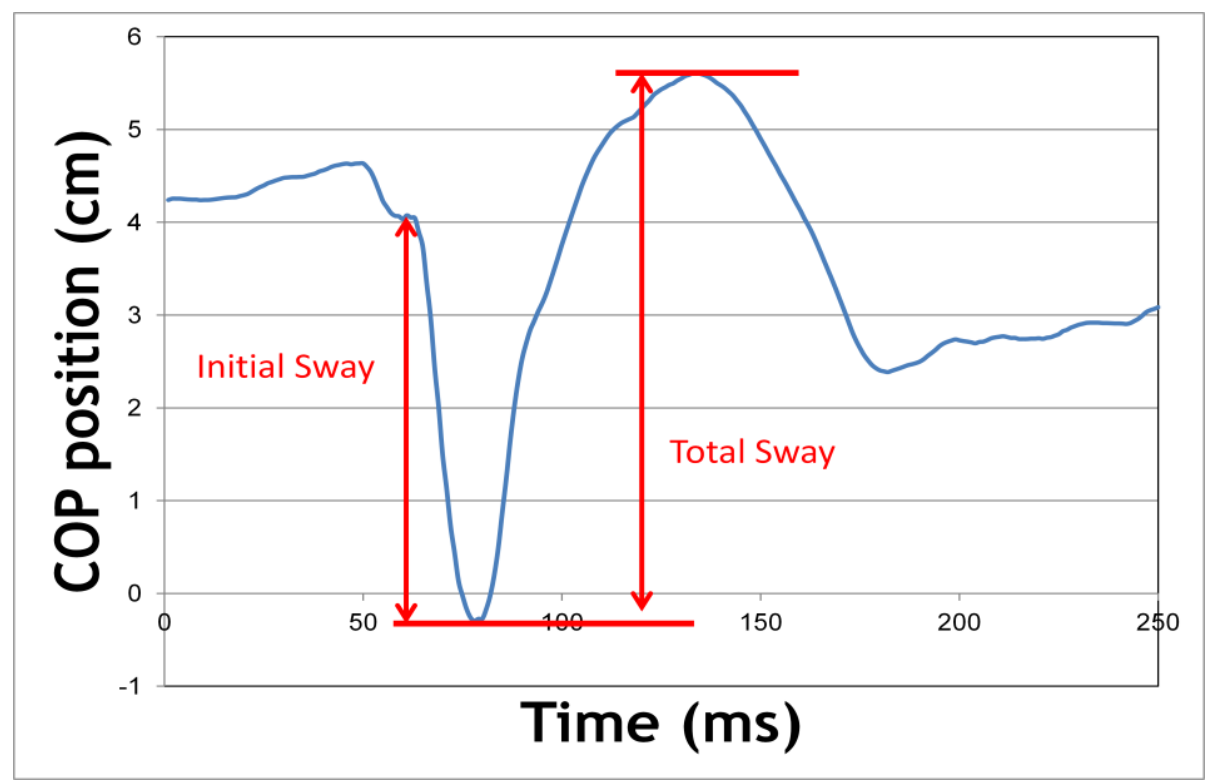

Figure 3: Graphical depiction of the initial sway and total sway variables that were obtained and calculated from the ADT

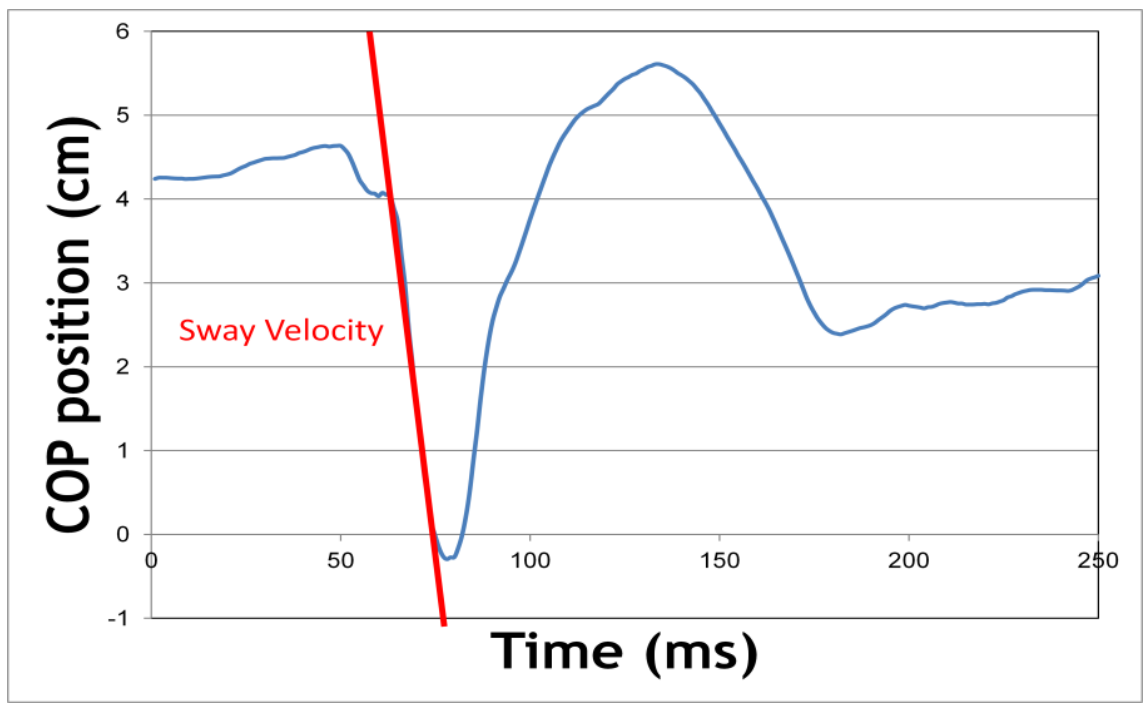

Figure 4: Graphical depiction of the sway velocity in a dynamic perturbation that was obtained and calculated from the ADT

The difference between the point at which surface rotation began (i.e. the commencement of the perturbation) and the point of maximum perturbation marked 
initial sway (Figure 3). Total sway was calculated by finding the difference between the maximum anterior sway and the maximum posterior sway COP displacements (Figure 3). Sway velocity in a dynamic perturbation was calculated by dividing the maximum perturbation by the difference between the time that the maximum perturbation occurred and the time of initial surface rotation (Figure 4).

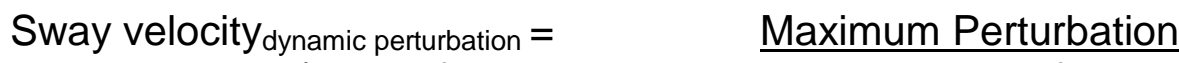

(Time of Maximum Perturbation - Time of Initial Surface Rotation)

\section{Post-Testing Urinary Incontinence Questionnaire}

Upon completion of the SOT and the ADT, the subjects were asked to fill out a brief subjective questionnaire about any urine leakage they experienced throughout the course of the testing. A numerical scale assessed the severity of any urine that was excreted. The Post-Testing Urinary Incontinence Questionnaire can be found in Appendix E.

\section{Statistical Analyses}

All of the static and dynamic variables, from the SOT and the ADT respectively, were calculated using Matlab (R2012a, MathWorks, Inc., Natick, MA). The statistical analyses were performed using the IBS SPSS software. Basic demographic descriptions of the control group and experimental group, including height, weight, mass, BMI, number of falls, and number of pregnancies were compared using an ANOVA; the alpha value was set at 0.05 (Table 1).

Specific Aim 1 was to examine if women with urinary incontinence demonstrate poorer postural stability during a static task (i.e. quiet standing) with two visual conditions (eyes opened and eyes closed) as compared to an age and parity matched control group. Since the variables of anterioposterior sway, mediolateral sway, length of 
the path of COP, sway velocity in quiet stance, and elliptical sway area all came from the same trial, they were not considered independent of each other. A 2x2 MANOVA was performed on each of these dependent variables; there were two groups (experimental and control) and also two visual conditions (eyes opened and eyes closed). The alpha value was set at 0.05 .

Specific Aim 2 was to examine if women with urinary incontinence demonstrate poorer postural stability during dynamic postural perturbations (i.e. toes up and toes down tilt of the support surface) as compared to an age and parity matched control group. It was assumed that since initial sway, total sway, and sway velocity in a dynamic perturbation were obtained from the same trial that they were not independent from one another. Therefore, a 2x2 MANOVA was performed on each dependent variable. There were two groups (experimental and control) and two directions of support surface tilt (toes up and toes down). The alpha value was set at 0.05 . 


\section{Chapter 4: Results}

Specific Aim 1: Static Postural Stability was to examine if women with urinary incontinence demonstrate poorer postural stability during a static task (i.e. quiet standing) during two visual conditions (eyes opened and eyes closed) as compared to an age and parity matched control group. Specifically, the variables of anterioposterior sway, mediolateral sway, length of the path of the center of pressure, sway velocity in quiet stance, and elliptical sway area, were examined during both the eyes opened and eyes closed tasks.

When comparing those with UI to those without, sway in both the anterioposterior and mediolateral directions were not significantly different $(p=0.987$ and $p=0.515$, respectively). Likewise, length of the path of center of pressure was not different between groups $(p=0.495)$. The sway velocity of the UI group was not different from the control group ( $p=0.495)$. Lastly, the two groups displayed no statistical differences in elliptical sway area $(p=0.254)$. In summary, based on the static variables assessed with the SOT, there were no significant differences noted when comparing the women with UI to those without UI. These findings are depicted in Table 2.

Table 2: Static postural stability variable totals (mean \pm standard deviation) between the control group and UI group.

\begin{tabular}{|c|c|c|c|}
\hline & Control & Ul Group & p-value \\
\hline Anterioposterior Sway $(\mathrm{mm})$ & $12.70 \pm 5.59$ & $12.70 \pm 6.10$ & 0.987 \\
\hline Mediolateral Sway $(\mathrm{mm})$ & $5.33 \pm 3.30$ & $4.83 \pm 2.54$ & 0.515 \\
\hline Length of COP Path $(\mathrm{mm})$ & $185.17 \pm 36.83$ & $178.31 \pm 62.23$ & 0.495 \\
\hline Sway Velocity $(\mathrm{mm} / \mathrm{sec})$ & $9.14 \pm 1.78$ & $8.89 \pm 3.05$ & 0.495 \\
\hline Elliptical Sway Area $\left(\mathrm{mm}^{2}\right)$ & $0.76 \pm 0.51$ & $1.27 \pm 1.52$ & 0.254 \\
\hline
\end{tabular}


Throughout the duration of the SOT, two visual conditions (i.e. eyes open and eyes closed) were assessed. Although not explicitly addressed in the specific aims or hypotheses, it was expected that subject performance in the eyes closed condition would reflect more sway (i.e. more instability) compared to the eyes open condition. In terms of anterioposterior sway $(p=0.001)$, length of the COP path $(p=<0.001)$, sway velocity $(p=<0.001)$, and elliptical sway area $(p=0.034)$, all subjects showed more sway when their eyes were closed. However, mediolateral sway was not different between visual conditions $(p=0.771)$. Table 3 gives the means, standard deviations, and $p$ values of all static variables when eyes were open and when eyes were closed.

Table 3: Static postural stability variables (mean \pm standard deviation) between the eyes open and eyes closed conditions experienced throughout the SOT.

\begin{tabular}{|c|c|c|c|}
\hline & Eyes Open & Eyes Closed & p-value \\
\hline Anterioposterior Sway $(\mathrm{mm})$ & $10.41 \pm 4.57$ & $14.73 \pm 6.10$ & $\mathbf{0 . 0 0 1}$ \\
\hline Mediolateral Sway $(\mathrm{mm})$ & $5.08 \pm 3.56$ & $4.83 \pm 2.29$ & 0.771 \\
\hline Length of COP Path $(\mathrm{mm})$ & $159.77 \pm 30.99$ & $203.71 \pm 57.66$ & $<\mathbf{0 . 0 0 1}$ \\
\hline Sway Velocity $(\mathrm{mm} / \mathrm{sec})$ & $7.87 \pm 1.52$ & $10.16 \pm 2.79$ & $<\mathbf{0 . 0 0 1}$ \\
\hline Elliptical Sway Area $\left(\mathrm{mm}^{2}\right)$ & $0.76 \pm 0.51$ & $1.27 \pm 1.52$ & $\mathbf{0 . 0 3 4}$ \\
\hline
\end{tabular}

Comparisons made between each group and visual condition showed no interactions; there are no significant differences to report. More specifically, anterioposterior sway $(p=0.161)$, mediolateral sway $(p=0.090)$, length of the path of $\operatorname{COP}(p=0.174)$, sway velocity $(p=0.174)$, and elliptical sway area $(p=0.347)$ were not significantly different between the control and UI group when taking into account whether eyes were opened or closed (Table 4). 
Table 4: Eyes open and eyes closed static postural stability variables (mean \pm standard deviation) for the control and UI groups.

\begin{tabular}{|c|c|c|c|c|c|}
\hline & \multicolumn{2}{|c|}{ Eyes Open } & \multicolumn{2}{c|}{ Eyes Closed } & \multirow{2}{*}{$\begin{array}{c}\text { p- } \\
\text { value }\end{array}$} \\
\hline & Control & UI group & Control & UI group & \\
\hline Anterioposterior & $11.43 \pm$ & $9.65 \pm$ & $13.97 \pm$ & $15.49 \pm$ & \\
Sway (mm) & 4.83 & 4.07 & 5.84 & 6.35 & 0.161 \\
\hline Mediolateral Sway & $5.84 \pm$ & $4.32 \pm$ & $4.57 \pm$ & $5.33 \pm$ & \\
(mm) & 4.32 & 2.03 & 1.52 & 2.79 & 0.090 \\
\hline Length of the Path & $170.18 \pm$ & $149.61 \pm$ & $200.15 \pm$ & $207.26 \pm$ & \\
of COP (mm) & 26.67 & 32.26 & 39.88 & 72.14 & 0.174 \\
\hline Sway Velocity & $8.64 \pm$ & $7.37 \pm$ & $9.91 \pm$ & $10.41 \pm$ & \\
(mm/sec) & 1.27 & 1.52 & 2.03 & 3.56 & 0.174 \\
\hline Elliptical Sway Area & $0.74 \pm$ & $0.76 \pm$ & $1.02 \pm$ & $1.52 \pm$ & \\
(mm ${ }^{2}$ ) & 0.51 & 0.76 & 0.76 & 1.78 & 0.347 \\
\hline
\end{tabular}

Specific Aim 2: Dynamic Postural Stability was to examine if women with urinary incontinence demonstrate poorer postural stability in response to dynamic postural perturbations (i.e. toes up and toes down tilt of the support surface) as compared to an age and parity matched control group. Specifically, the variables of initial sway, total sway, and sway velocity in a dynamic perturbation were assessed in response to both toes up and toes down perturbations.

The women with UI had less initial sway than the controls $(p=0.006)$. The UI group also had less total sway ( $p=0.008$, and less sway velocity $(p=0.001)$ (Table 5$)$.

Table 5: Dynamic postural stability variable totals (mean \pm standard deviation) between the control group and UI group.

\begin{tabular}{|c|c|c|c|}
\hline & Control & Ul Group & p-value \\
\hline Initial Sway $(\mathrm{mm})$ & $19.81 \pm 10.16$ & $15.75 \pm 9.40$ & $\mathbf{0 . 0 0 6}$ \\
\hline Total Sway $(\mathrm{mm})$ & $82.55 \pm 20.32$ & $72.64 \pm 25.65$ & $\mathbf{0 . 0 0 8}$ \\
\hline Sway Velocity $(\mathrm{mm} / \mathrm{s})$ & $89.15 \pm 42.16$ & $68.07 \pm 40.89$ & $\mathbf{0 . 0 0 1}$ \\
\hline
\end{tabular}


Further analysis of the initial sway, total sway, and sway velocity revealed that the subjects had greater sway when experiencing toes up perturbations compared to toes down. The specific $p$-values confirming these findings can be found in Table 6 .

Table 6: Dynamic postural stability variables (mean \pm standard deviation) between the toes up and toes down perturbations experienced throughout the ADT.

\begin{tabular}{|c|c|c|c|}
\hline & Toes Up & Toes Down & p-value \\
\hline Initial Sway $(\mathrm{mm})$ & $21.84 \pm 9.91$ & $13.72 \pm 8.38$ & $<\mathbf{0 . 0 0 1}$ \\
\hline Total Sway $(\mathrm{mm})$ & $84.84 \pm 22.86$ & $70.36 \pm 22.10$ & $<\mathbf{0 . 0 0 1}$ \\
\hline Sway Velocity $(\mathrm{mm} / \mathrm{s})$ & $97.03 \pm 39.12$ & $60.20 \pm .38 .1$ & $<\mathbf{0 . 0 0 1}$ \\
\hline
\end{tabular}

Initial sway across groups did not reflect any significant differences when taking into account perturbation direction $(p=0.821)$; sway velocity $(p=0.907)$ and total sway $(p=0.066)$ also reflected no group interaction. Table 7 details the group by direction analyses of initial sway, sway velocity, and total sway.

Table 7: Toes up and toes down dynamic postural stability variables (mean \pm standard deviation) for the control and UI groups.

\begin{tabular}{|c|c|c|c|c|c|}
\hline & \multicolumn{2}{|c|}{ Toes Up } & \multicolumn{2}{c|}{ Toes Down } & \multirow{2}{*}{ p-value } \\
\hline & Control & Ul group & Control & Ul group & -val \\
\cline { 1 - 5 } Initial Sway $(\mathrm{mm})$ & $23.88 \pm$ & $19.81 \pm$ & $16.00 \pm$ & $12.19 \pm$ & \\
\hline & 9.91 & 9.91 & 9.14 & 6.60 & 0.821 \\
\hline & $86.36 \pm$ & $83.31 \pm$ & $78.74 \pm$ & $61.72 \pm$ & \\
Total Sway $(\mathrm{mm})$ & 20.32 & 25.40 & 19.81 & 21.59 & 0.066 \\
\hline & $107.19 \pm$ & $86.87 \pm$ & $70.87 \pm$ & $49.28 \pm$ & \\
Sway Velocity $(\mathrm{mm} / \mathrm{s})$ & 39.62 & 36.58 & 37.08 & 36.32 & 0.907 \\
\hline
\end{tabular}


The questionnaires that each subject completed were not statistically analyzed, however the responses were used for subjective information. The subjects were asked about their clinical UI diagnosis that was then confirmed by one of the primary investigators (KT). Five women had stress incontinence, two had mixed incontinence, and no women reported urge incontinence.

Four of the subjects, all over the age of 52 , confirmed that they have already undergone menopause. Also, based on height and weight measurements, the subjects in the UI group varied in BMI classifications: normal weight $(n=3)$, overweight $(n=1)$, obese $(n=3)$. The control group was not matched based on BMI values, therefore the number of control subjects in each BMI category differed from those noted above for the experimental group: underweight $(n=1)$, normal weight $(n=5)$, obese $(n=1)$.

Since the two groups were paired based on parity, the number of pregnancies reported by the experimental subjects was the exact same as the number of pregnancies reported by the control subjects. Questions regarding number of pregnancies showed the following: two pregnancies $(n=3)$, one pregnancy $(n=3)$, and zero pregnancies $(n=1)$.

Five of the seven subjects affected by UI reported regular weekly exercise. Three subjects reported exercising three times per week, one subject two times per week, and one subject seven days per week. The most commonly reported exercise modality was walking $(n=5)$. Other types of exercise included: stretching, aerobics, bicycling, strength training, and elliptical usage. As for the control group, all seven subjects reported exercising at least 3 days per week. Specifically, four control subjects said they exercise three times per week, one subject four days, and two subjects five 
days. Similar to the UI group, the mostly commonly reported exercise modality was walking $(n=4)$. Aerobics, biking, ice skating, senior citizen fitness classes, strength training, running, and yoga were among other modalities specified.

According to the Balance and Falls Questionnaire, only one woman with UI reported falling; in fact, this subject noted five falls since the onset of her mixed UI symptoms. However, this particular subject also reported having problems with balance prior to her incontinence. On this same questionnaire, three subjects either "agreed" $(n=2)$ or "strongly agreed" $(n=1)$ that correcting any imbalance noticed since their first symptoms of UI would enhance their quality of life. 


\section{Chapter 5: Discussion}

This study proposed to examine the dynamic and static stability of women with stress, urge, or mixed incontinence. Our rationale was heavily based on the idea that women with UI have an increased rate of falling (6). Our primary goal was to understand the relationship between $\mathrm{UI}$ and postural instability. Additionally, both static and dynamic measures were examined in order to get a more complex assessment of stability. Our overall hypothesis was that women with UI would demonstrate more sway, evidenced by more COP movement, during static and dynamic stability testing, thereby revealing more instability.

\section{Specific Aim 1: Static Postural Stability}

Postural stability measures were acquired from COP data obtained from force plate readings. The SMART Balance Master was the primary method of obtaining stability data. More specifically, static postural stability was examined through the SOT that required each subject to stand quietly on a force plate with their eyes open, and then their eyes closed. The general hypothesis was that through many different variables, women with UI would show poorer stability in both the eyes open and eyes closed conditions. Chapter 1 specifically details each specific aim and corresponding hypotheses.

Our results refuted our hypotheses since no differences were found between the UI group and control group in any of the static stability variables. Even though our data did not show any differences, the SOT has been previously shown to be effective at detecting dissimilarities in stability among various groups of people. For instance, when specifically taking into account the eyes open and eyes closed parts of the SOT 
(condition 1 and condition 2, respectively), it was found that based on higher equilibrium indices, generally healthy adults ages 20 to 56 were more stable, under static conditions, when compared to older adults with and without vestibular dysfunction (59). This study by Pedalini et al. assessed the stability responses of several populations and the findings obtained from quiet standing conditions showed that the SOT has the capability of detecting stability differences between groups (59).

Because no static differences were determined between the $\mathrm{UI}$ and control groups in the current study, several factors need to be considered. For instance, a possible contributor to these findings is that subjects were not paired based on activity level. Yoo et al. suggest that a three month walking program has the capacity to decrease the risk of falling by changes reflected in body mass, aerobic endurance, upper body strength, and leg strength (60). However, this is inconclusive since it has been shown by Wolfson et al. that certain types of exercises, specifically lower extremity resistance training in conjunction with Tai Chi practices, did not change performance on the SOT (61). In the latter study, the number of times a subject lost their balance throughout each of the six SOT conditions (see chapter 3) was utilized as the measure of stability; the analyses focused on loss of balance (61) and not the same static variables that were calculated in our study.

Considering that our experimental and control subjects were not paired based on body weight, our hypotheses did not include predictions about differences in sway relative to weight. Based upon the calculation of subject demographics, we found that the UI group had a mean body mass of $79.38 \pm 19.45$ kilograms and the control group of $60.47 \pm 8.32$ kilograms. According to Hue et al., body weight and postural stability 
demonstrate an inverse relationship as measured through standing with eyes open and eyes closed on a force plate (21). This conflicts with our findings because the UI group had significantly more body weight $(p=0.036)$, but did not exhibit poorer stability compared to the controls. However, it is important to note that the study conducted by Hue et al. only included males in their analyses (21), so various physiological gender differences may have been contributing characteristics.

On the Balance and Falls Questionnaire, we did not include questions regarding perception of balance or fear of falling. Based on findings from Talkowski et al., the reasoning for the similarities in our static results might have been due to a lack of consideration of perceptual differences. Prior to completing a walking test, Talkowski et. al asked subjects to rate their perception of their own balance (62). It was found that those who perceived their balance as good, as opposed to discordant or poor, performed better on the walking test (62). Although this test did not directly measure balance, but instead walking distance, it provides knowledge of how psychological factors, such as perception, can contribute to performance (62). Additionally, the Activities-specific Balance Confidence (ABC) scale essentially assesses fear of falling in aging populations $(40,63)$. An association found between fear of falling, as obtained from the $A B C$ scale, and physical functioning contributes to the ways in which perception (i.e. fear) correlates to physical performance (63). Perhaps asking our subjects to rate their balance perception would have shown that women with UI perceive their balance as poor and therefore concentrate more on maintaining stability. Insight into balance perception could have exposed reasons why static stability was not different between our two groups. 
When not differentiating between women with $\mathrm{UI}$ and the controls, differences were found when measuring sway in the eyes open and eyes closed static conditions. We found that subjects in both groups had less sway when their eyes remained open. This supports findings from Chen et al. who reported that subjects without visual impairments had less sway versus subjects who had "low vision" or blindness (50). In other words when vision was inhibited, performance was negatively affected (50). The general findings from the study by Chen et al. are comparable to our study's findings, however the subject age demographics ( $\geq 70$ years old) differed from ours. Also their experimental group had health conditions that caused impaired vision, whereas simply closing the eyes challenged our subjects' vision. In another study examining static balance using the SOT, it was found that older adults demonstrated more postural imbalance when visual input was absent (64). Similarly, Grace-Gaerlan et al. used the SMART Balance Master protocols and pointed out that based on the assessment of various inputs of stability (i.e. visual, somatosensory, and vestibular systems), the visual system had the most imperative role in balance maintenance for healthy adults in their twenties and thirties (65).

It has been well established that aging populations are at an increased risk of falling $(11,20,22,23,51)$; falls are often reflective of instability (38). In a study by Choy et al., an unstable foam surface was placed on top of a force plate during the SOT; single-leg stance performance was also evaluated (20). Their main conclusion was that women 40 to 60 years old showed the most balance decrements on some of the tests (20). Our experimental and control subjects were paired based on age (+/- 3 years); the mean ages of the $U \mathrm{I}$ and control groups were $51.57 \pm 17.85$ and $51.71 \pm 18.20$, 
respectively. Most of the participants were within the 40 to 60 year old age range detailed by Choy et. al (20). Perhaps we saw no differences in the static variables because natural aging processes, such as hormonal alternations, that cause changes in stability were affecting these women similarly.

Lastly, Chiarelli et al. showed that older adults with urge incontinence experienced more falls compared to those with stress incontinence (6). Our results dispute this finding since only one of our UI subjects reported falling, but an underrepresentation of each different type of UI (i.e. stress, urge, and mixed) could be a reason why. The clinical UI diagnoses revealed that none of our subjects had urge incontinence.

\section{Specific Aim 2: Dynamic Postural Stability}

Dynamic measures of postural stability were obtained from force plate readings acquired from the ADT protocol of the SMART Balance Master. The subjects experienced toes up and toes down perturbations aimed at challenging their stability. The general hypothesis was that through many different variables, women with UI would have poorer dynamic stability, demonstrated by increased COP movement, despite the direction of the perturbation (i.e. toes up or toes down). Chapter 1 specifically details each specific aim and corresponding hypotheses.

Our results reflected a difference between groups and showed that initial sway, total sway, and sway velocity were all less in the UI group. Since it was our assumption that increased COP movement, and ultimately more sway, would reflect poorer postural stability, we believe that based on less COP movement, the UI group actually demonstrated more dynamic postural stability. Few studies have set out to investigate 
postural stability as it relates to $\mathrm{UI}(6,7,13,17)$ and none of them utilized the variables that we chose to calculate.

Smith et al. found that women with stress UI had delayed pelvic floor muscle activation (17). Because pelvic floor muscles have a suspected role in balance maintenance $(13,16)$, delayed activation may mean that these muscles have a compromised ability to readily perform their stability-related functions (17). Although Smith et al. (17) did not examine the same dynamic variables that we did, their results showing that rapid arm movements increased the time it took for pelvic floor muscles to become activated, provided some reasoning as to why we hypothesized women with UI to have poorer stability. But again, this was not the case and our hypotheses were not supported. Since our Ul group had less COP movement in reaction to the perturbations, our results disprove our predictions that instability would be a product of delayed pelvic floor muscle activation (17). It should be noted however that it is difficult to make concrete comparisons to the data obtained by Smith et al. because our means of assessment and variables obtained were dissimilar.

Previous literature has shown that certain postural muscles of healthy male and female subjects engage in feed-forward responses when balance is compromised by translational perturbations (66). More specifically, in response to backward perturbations, the timing of the activation measured in the soleus, rectus abdominis, and medial and lateral heads of the gastrocnemius coincided with one another (66). Similarly, simultaneous tibialis anterior and erector spinae activation during forward perturbations was reported (66). Such findings reveal that certain postural muscle activities are not individualized, but rather synchronized (66). In relation to our study, 
the significantly less amount of COP movement measured among the UI group could partly be due to a compensation mechanism presented as enhanced co-contractions of other muscles relevant to stability maintenance. Furthermore, challenges of stability might further stimulate the activation of other postural muscles to help supplement the characteristically weak pelvic floor muscles of women with UI (7). In fact, Smith et. al reported that as the activity of the pelvic floor muscles increased in response to a perturbation, external oblique activity also got larger (7). Additional evidence showing that compensation occurs would help provide more concrete rationale as to why we saw less COP movement in our experimental group.

Smith et al. also reported that challenging stability through dropping a one kilogram weight into a bucket held by the subjects resulted in increased pelvic floor muscle activity of women with incontinence (7). This increase could potentially provide even more evidence of compensatory adaptations experienced by UI-affected women. It is a possibility that our UI group had less COP movement on the dynamic testing due to an increased magnitude of pelvic floor muscle activity. In other words, increased muscle activity may be a way that women with $\mathrm{UI}$ have adapted to help maintain stability. The musculature of women with UI may have undergone adaptations (e.g. enhanced muscle activation and co-contractions) to counteract weak pelvic floor muscles and a potential vulnerability to falling; these adaptations would better enable them to maintain stability.

In a study assessing dynamic stability of pregnant women, initial sway, total sway, and sway velocity were all significantly less in the third trimester as compared to women in their second trimester and women who were not pregnant (53). Pregnant 
women are a population generally considered vulnerable to falling; it was expected that the further along a women was in her pregnancy, the more instability she would experience (53). However, the results from McCrory et al. did not coincide with this expectation (53). As it relates to our study, women with UI may be another group, similar to pregnant women, vulnerable to instability, who had less COP movement during perturbed stances.

In another study by McCrory et al., dynamic responses also measured by initial sway, total sway, and sway velocity were all significantly less in pregnant women who had fallen compared to a control group and pregnant women who had not reported any falls (52). Therefore, women who were assumed to have poorer stability, based on experiencing falls, did not have as much COP movement as the other groups.

Similarly, it was hypothesized that in response to computerized posturography limit of stability measures, sway velocity would be greater in subjects with chronic low back pain (67). However, contrary to this hypothesis, the subjects with chronic low back pain actually had less sway velocity in the forward direction compared to the control group (67). Davis et. al discussed that this may be a compensatory response resulting from fear of movement and reflecting a tactic to avoid pain (67).

In order to more thoroughly analyze why our UI group demonstrated less COP movement, typical COP movement should be considered. In a toes up perturbation, dorsiflexion of the ankle helps to activate the muscle spindles of the soleus; this activation leads to a stretch reflex of the plantar flexors that essentially causes the toes to press downward into the surface in an attempt to maintain stability (68). This entire series of events causes the COP to move anteriorly (68). A toes down perturbation can 
be detailed by similar processes and muscular reactions, but the COP would be expected to move in a posterior direction (68). In relation to the current study, muscular co-contraction may provide reasoning as to why the COP movement was less in our UI group; these subjects may be using a mechanism, different than the one detailed above, to maintain stability in the presence of perturbations.

To more thoroughly analyze COP and center of mass, it is important to take into consideration the mechanical relationship of these two factors, particularly as it relates to an individual standing on a force plate. During a weight-bearing stance, COP is the point at which the vertical ground reaction force is located and acts in an upward direction (69). According to Newton's Third Law, the force exerted by this ground reaction force and the force exerted by the body standing on the force plate are equal in magnitude and opposite in direction (69). More specifically, the body exerts a downward force that can be calculated by an equation detailed by Newton's Second Law: Force $=$ Mass $\times$ Acceleration .

A healthy population has been noted to utilize a "hip strategy" or "ankle strategy" in order to help sustain stability (70). Although it is difficult for the body to rely independently on the hips or independently on the ankles, one joint (i.e. one strategy) generally contributes more readily than the other to stability maintenance (70). Since we did not evaluate factors beyond COP movement, we are unsure if there was a difference between groups in the primary strategy used to maintain stability in the presence of each perturbation. However, such measurements could help to further explain the differing dynamic stability results we found. 
One last detail that is important to consider is that our study did not analyze differences in regular exercise participation between the two groups. In some cases, increased activity level, in terms of a regular walking exercise program, has been shown to improve certain factors that enhance stability (60). Since our study did not pair subject based on such variables, it is difficult to decipher whether or not the less initial sway, total sway, and sway velocity of the UI group was impacted by engaging in more physical activity.

\section{Limitations}

Before making general conclusions or extending these findings to the larger population, several limitations need to be considered. Of primary importance is the number of subjects. Each group only consisted of 7 subjects; a power analysis of the SOT variables revealed that a minimum of 60 subjects would be needed to show a difference in elliptical sway area and a minimum of approximately 500 subjects would be needed to show group differences in anterioposterior sway, mediolateral sway, length of the path of COP, and sway velocity. So although one of the biggest struggles encountered throughout the course of this study was subject recruitment, it is safe to conclude that sample size would not have made a difference in the SOT variable analyses.

Secondly, we can only assume that differences in dynamic stability represent differences in muscle contractions. So, because we did not include muscle EMG recordings in our methods, confirmation of this rationale is limited.

The criteria in which the control subjects were paired to the UI group may have influenced the results; activity level and weight were not considered. Other studies 
have found that exercise habits (60) and body weight (21) influence the body's ability to maintain its stability. Therefore, the differences found between initial sway, total sway, and sway velocity might have been confounded by factors outside of UI.

The utilization of only the SOT and the ADT protocols may have limited the results. Our methods do not necessarily represent real-life scenarios that may challenge balance. Also, several subjects complained that the placement of their feet, per Neurocom guidelines, was an "unnatural stance"; this discomfort may have contributed to imbalance upon perturbation as well.

\section{Future Research}

In order to quantify potential muscular adaptations of women with UI, it would be helpful for future studies to include various EMG readings to see if and where muscular co-contractions/compensations occur. More knowledge on why the UI group showed less COP movement may be more apparent if muscle activity is measured. This would also provide better understanding of any muscle contraction contributions to stability.

In normal healthy populations, COP movement can predict center of mass movement (71), but this has not been proven in a perturbed stance in women with UI. Therefore in future research, the inclusion of accelerometers on landmarks, such as the sacrum, during various stability tests would help indicate if center of mass movement follows COP movement in women affected by UI; an accelerometer could contribute additional information to the current study by adding reasoning as to why these women had less COP movement.

Fear of falling and perception of balance is important to include in future research studying stability and UI. Insight into how fearful the women with UI are of falling might 
positively correlate to the amount of muscular co-contraction that occurs when stability is challenged.

To obtain even more data from women with UI, dynamic and static postural stability should be assessed before and after physical therapy intervention. Future studies should focus on pre- and post-UI treatment to measure whether or not stability is different once UI symptoms have subsided (i.e. post-treatment).

Lastly, future research should include different balance testing protocols that will provide more static and dynamic balance-related data particularly relevant to everyday activities in which stability is essential.

\section{Conclusions}

It is difficult to directly compare our findings to those in the current literature since much of the Ul-related research focuses on how perturbations perpetuate symptoms such as urine excretion. Our study aimed to elaborate on the relationship between postural instability and a population of women affected by stress, urge, or mixed incontinence. Results from the SOT provided evidence that women affected by UI may not experience compromised static stability. However, some of our other results show that women with $\mathrm{UI}$ have less COP movement during dynamic stability testing. These findings obtained from the ADT coincide with some of the balance-related literature; in spite of pelvic floor muscle weakness, UI may promote certain muscular adaptations (e.g. increased activity and co-contraction) with the purpose of helping these women remain stable. As with any research, the limitations of the study should be kept in mind. Explanations for the enhanced dynamic stability of women with UI would be better supported through the incorporation of EMG readings in future studies. Supplemental 
research is needed to examine the direct influence of the pelvic floor and postural musculature on the stability of women affected by UI.

In conclusion, upon calculation of static variables we did not find any differences in COP movement between the UI group and the control group, but differences were evident when assessing dynamic stability. The static stances required during the SOT may not have challenged the balance of our subjects enough; this may be a main reason why differences were not measured. On the other hand, a test like the ADT presents more challenges to stability since perturbations occur. Therefore, in our opinion, the ADT may be a better predictor of true stability responses. 


\section{Appendix A: General Health Phone Screening Questionnaire}

Upon answering a phone call from a potential subject:

"Hello. Thank you for calling for information about the study. Let me tell you more about it so you can decide if you would like to participate. The purpose of the study is to determine if women with pelvic floor dysfunction, specifically urinary incontinence, demonstrate deficiencies in postural stability, in other words, balance.

As part of the study, we will also ask you to fill out a few brief questionnaires related to your experience with urinary incontinence and also related to balance and falls. You will also go through two different tests that will assess postural stability. The entire testing session, from the time you walk in the door until you are finished, should last approximately an hour and a half. Are you still interested in participating in the study?"

If the subject agrees, then proceed to the next paragraph:

"In order to determine if you are eligible to participate in the study, I need to ask you a series of questions. The questions will take approximately 10 minutes to ask. Is this okay? Please ask me about any questions that are not clear to you. "

\section{General Questions:}

1. What type of urinary incontinence have you been diagnosed with? Stress $\square \quad$ Urge $\square \quad$ Mixed $\square \quad$ Other $\square$ If subject answered "other", then subject is ineligible

2. What is your age? (If not at least 18 years old, subject is ineligible)

3. How much do you weigh?

4. How tall are you?

$$
\text { Use } 2 \text { and } 3 \text { to calculate BMI: }
$$

5. Do you currently take any medications? Yes $\square \quad$ No If subject answered "yes", then list here:

6. How many pregnancies have you had? List the dates of each delivery (i.e. month and year) as well as the type of delivery (e.g. vaginal, cesarean, etc.): 


\section{General Health:}

7. Are you pregnant? Yes $\square \quad$ No $\square$ If subject answered "yes", then subject is ineligible

8. Are you 3 months or less post-partum? Yes $\square \quad$ No $\square$ If subject answered "yes", then subject is ineligible

9. Have you ever had a hysterectomy procedure? Yes $\square$ No If subject answered "yes", then subject is ineligible

10. Do you have diabetes? Yes $\square \quad$ No $\square$

If subject answered "yes", then ask the following question:

Do you have to take insulin for it? Yes $\square \quad$ No $\square$ If subject answered "yes", then subject is ineligible

11. Do you have uncontrolled high blood pressure? Yes $\square \quad$ No $\square$ If subject answered "yes", then subject is ineligible

12. Do you have any musculoskeletal dysfunctions or lower extremity injuries that affect your gait or require limited load bearing? Yes $\square \quad$ No $\square$

If subject answered "yes", then subject is ineligible

13. Have you had back surgery within the past year? Yes $\square$ No If subject answered "yes", then subject is ineligible

14. Do you have any sort of balance disorder(s), persistent dizziness or vertigo?

Yes $\square \quad$ No $\square$

If subject answered "yes", then subject is ineligible

15. Do you have neurological disorder or any neuromuscular impairment? Yes $\square \quad$ No $\square$ If subject answered "yes", then subject is ineligible

16. Can you stand unassisted for an extended period of time? Yes $\square \quad$ No $\square$ If subject answered "no", then subject is ineligible

\section{Exercise Habits:}

17. Do you currently exercise? Yes $\square \quad$ No $\square$

a. How often? times/week

b. Duration of exercise session:

c. Which types of exercise apply to your normal exercise routine? (Circle all that apply to the subject)

\begin{tabular}{|l|l|l|l|l|l|}
\hline $\begin{array}{l}\text { Organized } \\
\text { Sports }\end{array}$ & Walking & Jogging/Running & Swimming & Bicycling & Skating \\
\hline Martial Arts & Tai Chi & Aerobics & Dancing & $\begin{array}{l}\text { Strength/Weight } \\
\text { Training }\end{array}$ & Yoga \\
\hline
\end{tabular}

Other - please describe: 
If subject is eligible to complete the study, state the following:

"You meet all of our criteria for including you in the study. Would you like to schedule a time for you to come into the lab to do the tests that I explained earlier?"

Proceed to scheduling the subject....

If subject is not eligible to complete the study, state the following:

"Based on your answer to the question about (state question topic here), you are not eligible to complete in the study. Thank you for calling about the study though. There may be future studies for which you are qualified. My paperwork from our phone conversation will be destroyed so that no one else will see your answers." 


\section{WWestVirginiaUniversity.}

Office of Research Compliance

CONSENT AND INFORMATION FORM

OMR ICF

Principal Investigator: McCrory, Jean

Department: MEDICINE - Exercise Physiology

Tracking Number: $\quad \mathrm{H}-24350$

Study Title:

Is postural stability compromised in women with urinary incontinence?

Co-Investigator(s):

Rondini, Samantha; Harrison, Kate; Mancinelli, Corrie; Thomas, Krystal

Sponsor

WVU Injury Research and Control Center

Contact Persons

In the event you experience any side effects or injury related to this research, you should contact Dr. McCrory at 304/293-0442. (After hours contact Dr. McCrory at 725/554-4955.)

If you have any questions, concerns, or complaints about this research, you can contact Dr. McCrory at 304/293-0442.

For information regarding your rights as a research subject, you may contact the Office of Research Compliance at 304/293-7073.

Introduction

In addition if you would like to discuss problems, concerns, have suggestions related to research, or would like to offer input about the research, contact the Office of Research Integrity and Compliance at 304293-7073.

\begin{tabular}{lllll}
\hline Tracking \#: & $\mathrm{H}-24350$ & Page 1 of 7 & & \\
Approved On: & $10 / 20 / 2012$ & & Initials & \\
Valid Through: & $10 / 19 / 2013$ & & & \\
Last Amended: & $06 / 25 / 2013$ & &
\end{tabular}


You, , have been asked to participate in this research study, which has been explained to you by . This study is being conducted by Dr. Jean

McCrory, PhD. and Samantha Rondini, BS in the Department of Exercise Physiology at West Virginia University. This study is being conducted as part of Samantha Rondini 's Thesis requirements for completion of her Masters degree in Exercise Physiology.

Purposes of the Study

The purpose of this study is to examine the relationship between urinary incontinence and postural stability (i.e. balance). We are specifically looking for women with stress incontinence (urine leakage following an event such as a cough, sneeze, or in physical activity), urge incontinence (an unexpected released of a large amount of urine), or a combination of stress and urge incontinence. The primary goal of this research study is to provide clinicians and physical therapists information on balance being associated with urinary incontinence in women. The information gained from this study may be valuable in the treatment, or even prevention, of balance issues related to urinary incontinence. You have been invited to participate in this research study which involves assessment of balance. WVU expects to enroll approximately 10 subjects.

\section{Description of Procedures}

This study involves assessment of postural stability (i.e. balance). This study will require you to come in for testing on two occasions. During this time, you will complete a Balance and Falls Questionnaire, Urinary Incontinence Questionnaire, balance testing (Sensory Organization Test and Adaptation Test), and Post-Testing Urinary Incontinence Questionnaire. The completion of all components of this study will take approximately 1.5 to 2 hours each visit. You do not have to answer all the questions on the questionnaires. You will have the opportunity to see the questionnaires before signing this consent form.

Balance and Falls Questionnaire: You will be asked to fill out a questionnaire regarding any noticeable balance discrepancies and/or falls that you have experienced since the onset of your urinary incontinence symptoms. This will take approximately 10 to 15 minutes to complete. You

\begin{tabular}{lll}
\hline Tracking \#: & H-24350 & Page 2 of 7 \\
Approved On: & $10 / 20 / 2012$ & \\
Valid Through: & $10 / 19 / 2013$ & \\
Last Amended: & $06 / 25 / 2013$ &
\end{tabular}

$\overline{\text { Initials }} \overline{\text { Date }}$


do not have to answer all of the questions.

Urinary Incontinence Questionnaire: You will be asked to fill out a questionnaire related to your experiences with urinary incontinence and urination. Topics covered on this questionnaire include, but are not limited to, menses, menopause, and childbirth. There will also be some questions related to medical, social/psychological and exercise histories. This will take about 15-20 minutes to complete. You do not have to answer all of the questions.

Sensory Organization Test (SOT): Your static balance (balance while standing still) will be measured using a machine called the SMART Balance Master. You will be required to wear a harness as a safety precaution throughout the entire testing duration. You will stand barefoot on a platform and undergo 6 different conditions. Each condition will be repeated for 3 trials and each trial will last about 20 seconds. In each condition, the goal is to remain as still as possible despite what is happening around or underneath you. The conditions include: 1 . standing with your eyes open, 2 . standing with your eyes closed, 3 . eyes open, but the visual surroundings around you will be in motion, 4 . eyes open, but the platform beneath your feet will slightly rotate, 5 . eyes closed, but the platform beneath your feet will slightly rotate and 6 . eyes open, but the visual surroundings around you will be in motion and the platform beneath your feet will slightly rotate. Before each of the 6 conditions begin, you will be given verbal explanations of what will be happening throughout that specific part of the test. This testing will take approximately 20 minutes to complete.

Adaptation Test (ADT): Your dynamic balance (ability to maintain balance despite movements aimed at challenging it) will also be measured on the SMART Balance Master. You will be required to wear a harness as a safety precaution throughout the entire testing duration. You will stand barefoot on a platform and undergo 10 different trials. Each trial will consist of either a toes up or toes down rotation of the platform that you are standing on. During all of the rotations, the goal is to remain as still as possible despite what is happening underneath you. The first five trials will be toes up rotations followed by five toes down rotations. Before you start, you will be given a verbal explanation of what will be happening throughout this

$\begin{array}{lll}\text { Tracking \#: } & \text { H-24350 } & \text { Page } 3 \text { of } 7 \\ \text { Approved On: } & 10 / 20 / 2012 & \\ \text { Valid Through: } & 10 / 19 / 2013 & \\ \text { Last Amended: } & 06 / 25 / 2013 & \end{array}$

$\overline{\text { Initials }} \overline{\text { Date }}$


Tracking \#:

test. This testing will take approximately 15 minutes to complete.

Post-Testing Urinary Incontinence Questionnaire: Upon completion of both the SOT and the ADT, you will be asked to fill out a brief questionnaire about any urine leakage you experienced throughout the course of the testing. This will take 5-10 minutes to complete. You do not have to answer all of the questions.

If you an experimental subject, once you have completed your physical therapy treatment, you will be asked to come back to the Balance and Falls Lab to repeat all testing.

If you are a control subject, you will be asked to come back to the Balance and Falls Lab in approximately 6 weeks to repeat all testing.

Risks and Discomforts

Balance Testing (SOT and ADT): Due to the nature of the SOT, the support surface you will be standing on will slightly rotate and the visual surroundings will move during some of the conditions. During the ADT, the surface you will be standing on will move. These movements during both the SOT and the ADT may cause you to become startled when they begin, as well as potentially result in loss of balance and/or falling into the harness. In the instance of a fall such that you need the harness, minor injuries, soreness, and pain could result. If you are claustrophobic you may feel uncomfortable being within the confines of the SMART Balance Master. In order to your ensure safety, you will be required to wear a harness to attenuate any falls and to help in the prevention of any fall-related injuries. Additionally, an exercise physiologist who is familiar with emergency procedures will continuously monitor all testing.

Your current academic status or employment at WVU will not be impacted by your involvement in this study.

\section{Alternatives}

You do not have to participate in this study.

$\begin{array}{lll}\text { Tracking \#: } & \mathrm{H}-24350 & \text { Page } 4 \text { of } 7 \\ \text { Approved On: } & 10 / 20 / 2012 & \\ \text { Valid Through: } & 10 / 19 / 2013 & \\ \text { Last Amended: } & 06 / 25 / 2013 & \end{array}$

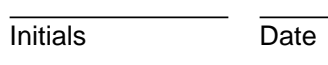


Due to the nature of the balance testing and the protocols required, there are no alternatives to the testing procedures.

Benefits

You may receive no benefit from participation in this study. The data will help researchers, exercise physiology practitioners, and physical therapists understand the relationship between urinary incontinence and postural stability. Additional benefits from your participation include insight into your static and dynamic postural stability status, in other words your balance. This information may be used to better understand some components of your overall health status and wellness.

Financial Considerations

You will receive $\$ 50.00$ for participating in this study; this will be awarded upon completition of each of the two testing visits. You may wish to consult your insurance carrier prior to entering this study. You will incur the costs of travel to the Health Science Center of West Virginia University School of Medicine for the testing session. There are no special fees for participating in this study, but any expense associated with injury or treatment of side effects will be billed to you or to your insurance company. If you are injured as a result of this research, treatment will be available.

Responsibility for this treatment will be borne by you and your insurance company. Compensation for your injuries will not be provided voluntarily by the investigator, West Virginia University or other associated affiliates.

Confidentiality

Any information about you that is obtained as a result of your participation in this research will be kept as confidential as legally possible. Your records and test results, just like hospital records, may be subpoenaed by court order or may be inspected by federal regulatory authorities without your additional consent. In addition, there are certain instances where the researcher is legally required to give information to appropriate authorities. In any publications that result from this research, neither your name nor any information from which you might be identified will be published without your consent.

Voluntary Participation

$\begin{array}{lll}\text { Tracking \#: } & \mathrm{H}-24350 & \text { Page } 5 \text { of } 7 \\ \text { Approved On: } & 10 / 20 / 2012 & \\ \text { Valid Through: } & 10 / 19 / 2013 & \\ \text { Last Amended: } & 06 / 25 / 2013 & \end{array}$

$\overline{\text { Initials }} \overline{\text { Date }}$


Participation in this study is completely voluntary. You are free to withdraw your consent to participate in this study at any time. Refusal to participate or withdrawal will not affect your future care, your class standing or grades or your employee status at West Virginia University, and will involve no penalty to you. In the event new information becomes available that may affect your willingness to participate in this study, this information will be given to you so that you can make an informed decision about whether or not to continue your participation. You have been given the opportunity to ask questions about the research, and you have received answers concerning areas you did not understand.

$\begin{array}{lll}\text { Tracking \#: } & \mathrm{H}-24350 & \text { Page } 6 \text { of } 7 \\ \text { Approved On: } & 10 / 20 / 2012 & \\ \text { Valid Through: } & 10 / 19 / 2013 & \\ \text { Last Amended: } & 06 / 25 / 2013 & \end{array}$


Upon signing this form, you will receive a copy.

I willingly consent to participate in this research.

\begin{tabular}{llll}
\hline $\begin{array}{l}\text { Signature of Subject or } \\
\text { Subjects Legal Representative }\end{array}$ & Date & & Timed Name
\end{tabular}

The participant has had the opportunity to have questions addressed. The participant willingly agrees to be in the study.

Signature of Investigator or

Printed Name

Date

Time

Co-Investigator

$\begin{array}{lll}\text { Tracking \#: } & \mathrm{H}-24350 & \text { Page } 7 \text { of } 7 \\ \text { Approved On: } & 10 / 20 / 2012 & \\ \text { Valid Through: } & 10 / 19 / 2013 & \\ \text { Last Amended: } & 06 / 25 / 2013 & \end{array}$

$\overline{\text { Initials }} \quad \overline{\text { Date }}$




\section{Appendix C: Balance and Falls Questionnaire}

1. Have you had any previous problems with balance prior to your symptoms of urinary incontinence? Yes $\square \quad$ No $\square$

a) If yes, what problems?

Please answer questions 2-5 as they relate to the time since you first noticed your symptoms of urinary incontinence...

2. How frequently do you lose your balance? (Circle one)

Often Occasionally Seldom Never

3. Are there any everyday activities in particular that challenge your balance?

Yes $\square \quad$ No $\square$

If yes, please describe.

4. Approximately how many falls have you experienced since you first noticed your symptoms of urinary incontinence?

5. Have you experienced any loss of balance resulting in a fall where some part of your body - other than your foot (or feet) - touched the ground?

Yes $\square \quad$ No $\square$

If 'yes', please respond to parts $\mathrm{a}-\mathrm{j}$ below as it relates to your most recent fall:

a) Date and time of fall:

b) Location of fall (e.g. home, work, etc.):

c) What were you doing at the time of the fall? (Circle any that apply)

I. Carrying an object or child

II. Turning, reaching or bending

III. Pushing, pulling or lifting

IV. Hurried pace

V. Running

VI. Other - please describe: 
d) Did you fall from an elevation greater than 3 feet? Yes $\square \quad$ No

e) What shoes were you wearing at the time of the fall (e.g. sneakers, heels, boots, etc.)?

f) Did you sustain an injury? Yes $\square \quad$ No $\square$

g) Were you ill at the time of the fall (e.g. hypoglycemia, nausea, vomiting, diarrhea, etc.)? Yes $\square \quad$ No

h) Were you performing a new task or unfamiliar task at the time of the fall? Yes $\square \quad$ No $\square$

i) If the fall occurred at work, was the fall reported to your employer? $\quad$ Yes $\square \quad$ No $\square$

j) Was a workers' compensation claim filed as a result of the fall? Yes $\square \quad$ No $\square$

6. If you have experienced challenges in your balance and/or have fallen since the onset of your urinary incontinence symptoms, on a scale of 0 to 10 , how does this affect your everyday life? (Circle one number)

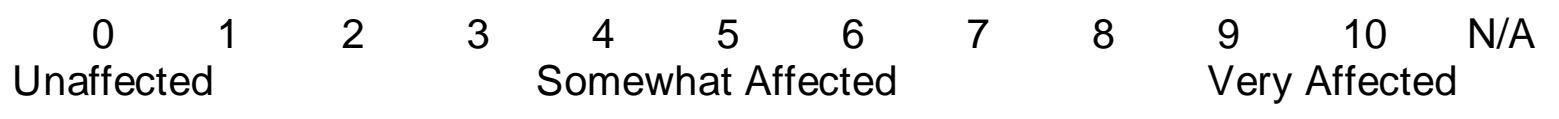

7. Please respond to the following statement by circling one of the options below: If you have experienced challenges in your balance and/or have fallen since the onset of your urinary incontinence symptoms, correcting these issues would enhance the quality of your life.

Strongly Disagree Disagree

Agree Strongly Agree

$\mathrm{N} / \mathrm{A}$ 


\section{Appendix D: Urinary Incontinence Questionnaire}

Beyond Kegels Assessment-History Adapted from Hulme, J. A. Beyond kegels book ii. Missoula: Phoenix Publishing; 1998

\begin{tabular}{llll}
\hline NAME & & \multicolumn{2}{c}{ DATE } \\
\hline SEX & AGE & BD & PHYSICIAN
\end{tabular}

\section{CHIEF COMPLAINT/PROBLEM}

Describe, state onset date, precipitating event, pattern of increased/decreased symptoms, previous treatment

$\overline{ } \overline{ }$

\section{SYMPTOM PICTURE}

\section{SYMPTOM DURATION}

yes no

Symptoms chronic ( $>3$ months)

Symptoms acute

Describe:

\begin{tabular}{|c|c|}
\hline DAYTIME TOILETING & yes \\
\hline Toileting every 4 hours & 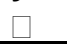 \\
\hline Toileting every $2-3$ hours & $\square$ \\
\hline Toileting every hour & $\square$ \\
\hline Toileting every 30-59 minutes & $\square$ \\
\hline Other & $\square$ \\
\hline
\end{tabular}

\begin{tabular}{|c|c|c|}
\hline NIGHTTIME TOILETING & yes & no \\
\hline Rarely/never & $\square$ & $\square$ \\
\hline Once a night & $\square$ & $\square$ \\
\hline Two to three times/night & $\square$ & ㅁ \\
\hline More than three times/night & $\square$ & \\
\hline Other & $\square$ & \\
\hline
\end{tabular}

LEAKING URINE NIGHTTIME yes no

Rarely/never

One to two nights/week

Three to four nights/week

More than four nights/week

Other

Describe:

\section{LEAKING URINE DAYTIME yes no}

Once every two weeks

Once a week

Two to three days/week

Four or more days/week

Once a day

Multiple times daily

Constantly all day

Other

Describe:

Morning primarily Afternoon/Evening primarily

No pattern

\section{Describe}

AMOUNT OF URINE LEAKED yes no

A few drops

A small gush or spurt

A large leak

Varies

Other

Describe:

PROTECTION USED [IF USED] yes no

Adult continence products (\#/day

Sanitary pads (\#/day

Pantiliner (\#/day

Other

Describe:

\section{ACTIVITIES RELATED TO LEAKING}

\begin{tabular}{lcc} 
Coughing/sneezing & yes & no \\
\hline Laughing & $\square$ & $\square$ \\
\hline Walking & $\square$ & $\square$ \\
\hline Position change & $\square$ & $\square$ \\
\hline Supine to sit & $\square$ & $\square$ \\
\hline Sit to stand & $\square$ & $\square$ \\
\hline Bending/lifting & $\square$ & $\square$ \\
\hline Running/jumping & $\square$ & $\square$ \\
\hline Aerobics & $\square$ & $\square$ \\
\hline Water running/shower & $\square$ & $\square$ \\
\hline Feeling cold & $\square$ & $\square$ \\
\hline During intercourse & $\square$ & $\square$ \\
\hline Before/during menstruation & $\square$ & $\square$ \\
\hline Key in the door & $\square$ & $\square$
\end{tabular}


When constipated

Other

Describe:

PERCEPTION OF NEED TO URINATE

yes no

No perception of bladder fullness

Leaks immediately after awareness

Leaks 1-2 min. after awareness

Toileting awareness without problem

Other

Describe:

\section{OBSERVATIONS DURING URINATION \\ yes no}

\begin{tabular}{ll} 
Difficulty initiating stream & $\square$ \\
\hline Weak/slow urine stream & $\square$ \\
\hline Dribbling after stream ends & $\square$ \\
\hline Pain during urination & $\square$ \\
\hline Burning during urination & $\square$ \\
\hline Blood in urine & $\square$ \\
\hline Abnormal color & $\square$ \\
\hline Abnormal odor & $\square$ \\
\hline Other $\quad \square$ Describe: & $\square$ \\
\hline
\end{tabular}

\section{BOWEL PATTERNS}

yes no

Experience frequent diarrhea

Experience frequent constipation

Bowel movements daily

Bowel movement every $2-3$ days

Bowel movement every 4-5 days
Use laxatives

Other

Describe:

\section{FLUID INTAKE}

yes no

Caffeine consumption (\#cups/day ) $\square \quad \square$

Alcohol intake (\#drinks/day )

Fluid consumption $<6-8$ glass/day Describe pattern of fluid intake:

\section{MEDICATION INTAKE}

yes no

Over the counter medication

Prescription medication

Diuretics

List:

\begin{tabular}{|c|c|c|}
\hline LIST SURGERIES & yes & no \\
\hline Bowel/Bladder & $\square$ & $\square$ \\
\hline Reproductive organs & $\square$ & $\square$ \\
\hline Spine/Back & $\square$ & $\square$ \\
\hline Brain & $\square$ & $\square$ \\
\hline Other & $\square$ & $\square$ \\
\hline \multicolumn{3}{|l|}{ Describe: } \\
\hline MEDICAL HISTORY & yes & no \\
\hline Neurological & $\square$ & $\square$ \\
\hline CNS conditions & $\square$ & $\square$ \\
\hline PNS conditions & $\square$ & $\square$ \\
\hline Musculoskeletal conditions & $\square$ & $\square$ \\
\hline Urinary tract infections & $\square$ & $\square$ \\
\hline Abdominal/pelvic pain & $\square$ & $\square$ \\
\hline Other & $\square$ & $\square$ \\
\hline
\end{tabular}

Describe:

\section{EXERCISE HISTORY}

yes no

Daily

5-6 times/week

3-4 times/week

1-2 times/week

None

Other

Describe type and duration

SOCIAL/PSYCHOLOGICAL HISTORY

yes no

Has leaking affected your lifestyle Childhood nighttime bedwetting

Pertinent family history - leaking

Abuse/injury

Other

Describe

\section{ACTIVITIES OF DAILY LIVING}

LIMITATIONS

NEEDS ASSISTANCE TO: yes no

Transport self to toilet

Transfer onto toile

Manage clothing

Describe:

\section{WOMEN ONLY}

PREGNANCY [\#

Vaginal deliveries (\# 


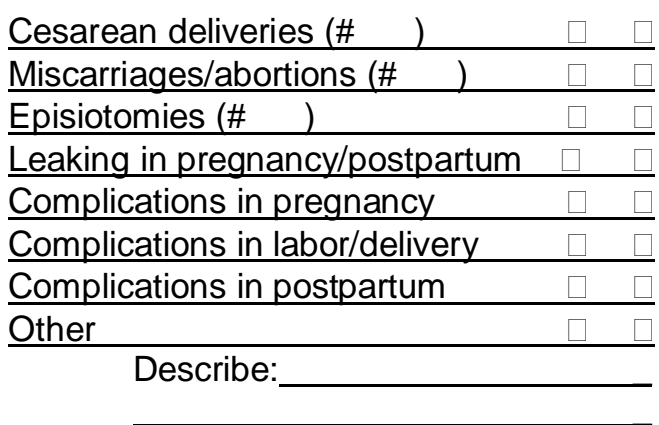

\section{MENSTRUATION [AGE ] yes no}

Change in urine control

Change in weight

Change in exercise habits

Change in overall health

Change in pain complaints

Other

Describe:

\section{MENOPAUSE [DATEIAGE \\ ] \\ yes no}

Change in urine control

Change in weight

Change in exercise habits

Change in overall health

Change in pain complaints

Other

Describe:

\section{PROBLEM LIST}

List the pertinent items checked in the yes column

1.

$\square \quad \square$

$\square \quad \square$

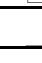

\section{$\square$}

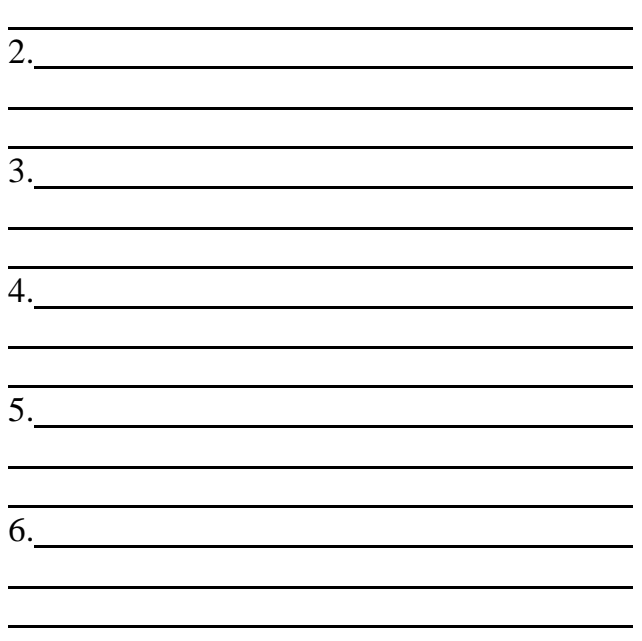

2

3

4

.




\section{Appendix E: Post-Testing Urinary Incontinence Questionnaire}

Now that you have completed the Sensory Organization Test and the Adaptation Test, please circle the number that corresponds to the urinary leakage that you experienced during the testing.

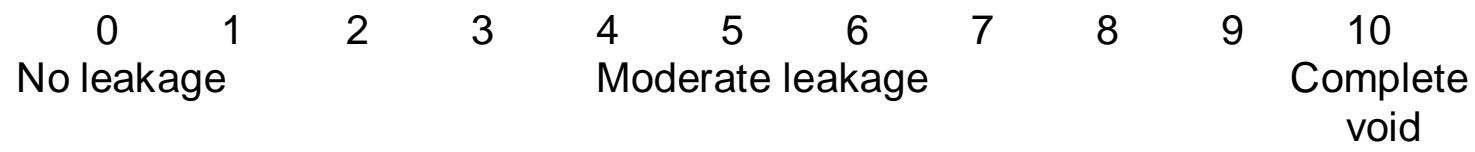

If you circled a number other than ' 0 ', was the leakage that you experienced during testing representative of the leakage that you normally experience?

\footnotetext{
Yes $\square \quad$ No $\square$

If you responded 'no', was the amount of urine less or more compared to the normal amount of leakage you experience?

Less $\square \quad$ More $\square$
} 


\section{References}

1. National Institutes of Health. Roughly one quarter of U.S. women affected by pelvic floor disorders. Retrieved from http://www.nih.gov/news/health/sep2008/nichd-17.htm. Septmeber 2008.

2. Wu JM, Hundley AF, Fulton RG, Myers ER. Forecasting the prevalence of pelvic floor disorders in U.S. women, 2010 to 2050. Obstet Gynecol 2009; 114: 127883.

3. MacLennan AH, Taylor AW, Wilson DH, Wilson D. The prevalence of pelvic floor disorders and their relationship to gender, age, parity and mode of delivery. BJOG 2000; 107:1460-70.

4. Hunskaar S, Burgio K, Diokno A, Herzog AR, Hjälmås K, Lapitan MC. Epidemiology and natural history of urinary incontinence in women. Urology 2003; 62(4 Suppl 1): 16-23.

5. Nygaard IE, Thompson FL, Svengalis SL, Albright JP. Urinary incontinence in elite nulliparous athletes. Obstet Gynecol. 1994;84: 183-7. Erratum in: Obstet Gynecol 1994; 84: 342.

6. Chiarelli PE, Mackenzie LA, Osmotherly P.G. Urinary incontinence is associated with an increase in falls. Aust J Physiother. 2009; 55: 89-95.

7. Smith MD, Coppieters MW, Hodges PW. Postural response of the pelvic floor and abdominal muscles in women with and without incontinence. Neurourology and Urodynamics 2007; 26: 377-385.

8. Arab AM, Chehrehrazi M. The response of the abdominal muscles to pelvic floor muscle contraction in women with and without stress urinary incontinence using ultrasound imaging. Neurourology and Urodynamics. 2011; 30: 117-120.

9. Mancinelli C, Mandich M, Utzman R. Preventing Falls in Community Dwelling Older Adults with Visual Impairment. Insight: Research and Practice in Visual Impairment and Blindness. 2011; 4: 124-132.

10. Chaudhry H, Findley T, Quigley KS, Bukiet B, Ji Z, Sims T, Maney M. Measures of postural stability. J Rehabil Res Dev 2004; 41: 713-20.

11. Prieto TE, Myklebust JB, Myklebust BM. Characterization and modeling of postural steadiness in the elderly: A review. IEEE Trans Rehabil Eng 1993; 1: 26-34. 
12. National Kidney and Urologic Diseases Information Clearinghouse. Urinary Incontinence in Women. Retrieved from http://kidney.niddk.nih.gov/kudiseases/pubs/uiwomen/UI-Women_508.pdf. October 2007.

13. Hodges PW, Sapsford, R, Pengel LHM. Postural and respiratory functions of the pelvic floor muscles. Neurourology and Urodynamics 2007; 26: 362-371.

14. Phillips SF, Edwards DA. Some aspects of anal continence and defaecation. Gut 1965; 6:396-406.

15. Howard D, Miller JM, Delancey JO, Asthon-Miller JA. Differential effects of cough, valsalva, and continence status on vesical neck movement. Obstet Gynecol 2000 95: 535-40.

16. Gosling JA, Dixon JS, Critchley HO, Thompson SA. A comparative study of the human external sphincter and periurethral levator ani muscles. Br J Urol 1981; 53: $35-41$.

17. Smith MD, Coppieters MW, Hodges PW. Postural activity of the pelvic floor muscles is delayed during rapid arm movements in women with stress urinary incontinence. Int Urogynecol J 2007; 18:901-911.

18. Bump RC, Hurt WG, Fantl JA, Wyman JF. Assessment of Kegel pelvic muscle exercise performance after brief verbal instruction. Am J Obstet Gynecol. 1991: 165: 322-329.

19. Glazer HI, Laine CD. Pelvic floor muscle biofeedback in the treatment of urinary incontinence: A literature review. Applied Psychophysiology and Biofeedback 2006; 31: 187-201.

20. Choy NL, Brauer S, Nitz J. Changes in postural stability in women aged 20 to 80 years. J Gerontol A Biol Sci Med Sci. 2003; 58: 525-30.

21. Hue O, Simoneau M, Marcotte J, Berrigan F, Dore J, Marceau P, Marceau S, Tremblay A, Teasdale N. Body weight is a strong predictor of postural stability. Gait Posture 2007; 26: 32-38.

22. Konrad HR, Girardi M, Helfert R. Balance and aging. Laryngoscope. 1999; 109:1454-60.

23. Rubenstein LZ. Falls in older people: epidemiology, risk factors and strategies for prevention. Age and Ageing 2006; 35(Suppl 2): ii37-ii41.

24. Marieb EN, Hoehn K. Human anatomy \& physiology, 7th edn. San Francisco: Pearson Benjamin Cummings, 2007. pp.350-351. 
25. Ashton-Miller JA, Delancey JO. Functional anatomy of the female pelvic floor. Ann N Y Acad Sci 2007; 1101: 266-296.

26. MacLennan $\mathrm{AH}$. The role of the hormone relaxin in human reproduction and pelvic girdle relaxation. Scand J Rheumatol 1991; (Suppl 88): 7-15.

27. Thorp JM Jr, Norton PA, Wall LL, Kuller JA, Eucker B, Wells E. Urinary incontinence in pregnancy and the puerperium: a prospective study. Am J Obstet Gynecol 1999; 181: 181:266.

28. Whitehead WE, Borrud L, Goode PS, Meikle S, Mueller ER, Tuteja A, Weidner A, Weinstein $M, Y e$ W. Fecal incontinence in US adults: epidemiology and risk factors. Gastroenterology. 2009; 137: 512-517.

29. National Digestive Diseases Information Clearinghouse. Fecal Incontinence. Retrieved from http://digestive.niddk.nih.gov/ddiseases/pubs/fecalincontinence/Fecal_Incontinen ce_508.pdf. April 2012.

30. Handa VL, Zyczynski HM, Burgio KL, Fitzgerald MP, Borello-France D, Janz NK, Fine PM, Whitehead W, Brown MB, Weber AM. The impact of fecal and urinary incontinence on quality of life 6 months after childbirth. Am J Obstet Gynecol 2007; 197: 636.

31. Kim CM, Jeon MJ, Chung DJ, Kim SK, Kim JW, Bai SW. Risk factors for pelvic organ prolapse. Int J Gynaecol Obstet 2007; 98: 248-251.

32. Bump RC, Mattiasson A, Bo K, Brubaker LP, DeLancey JO, Klarskov P, Shull $\mathrm{BL}$. The standardization of terminology of female pelvic organ prolapse and pelvic floor dysfunction. Am J Obstet Gynecol . 1996; 175:10-17.

33. Kelvin FM, Hale DS, Maglinte DDT, Patten BJ, Benson JT. Female pelvic organ prolapse: diagnostic contribution of dynamic cystoproctography and comparison with physical examination. AJR Am J Roentgenol 1999; 173: 31-37.

34. Kelvin FM, Maglinte DDT. Dynamic cystoproctography of female pelvic floor defects and their interrelationships. AJR Am J Roentgenol 1997; 169: 769.

35. Olsen, A.L., Smith VJ, Bergstrom JO, Colling JC, Clark AL. Epidemiology of surgically managed pelvic organ prolapse and urinary incontinence. Obstet.Gynecol 1997; 89: 501-506.

36. Sakakibara et al. Urinary function in elderly people with and without leukoaraiosis: relation to cognitive and gait function. J Neurol Neurosurg Psychiatry 1999;67:658-660. 
37. Hemborg B, Moritz $U$, Löwing, $H$. Intra-abdominal pressure and trunk muscle activity during lifting. IV. The causal factors of the intra-abdominal pressure rise. Scandinavian Journal of Rehabilitation Medicine 1985; 17: 25-38.

38. Piirtola M, Era P. Force platform measurements as predictors of falls among older people- a review. Gerontology. 2006; 52:1-16.

39. Mancini M, Horak FB. The relevance of clinical balance assessment tools to differentiate balance deficits. Eur J Phys Rehabil Med 2010; 46: 239-48.

40. Powell LE, Myers AM. The activities specific balance confidence (ABC) scale. $J$ Gerontol Med Sci 1995; 50: M28-34.

41. Horak FB. Clinical assessment of balance disorders. Gait Posture 1997; 6: 7684.

42. Thrane $\mathrm{G}$, Joakimsen RM, Thornquist $\mathrm{E}$. The association between timed up and go test and history of falls: The Tromsø study. BMC Geriatrics. 2007; 7.

43. Horak FB, Wrisley DM, Frank J. The Balance Evaluation Systems Test (BESTest) to differentiate balance deficits. Phys Ther 2009; 89: 484-498.

44. Lord SR, Clark RD. Simple physiological and clinical tests for the accurate prediction of falling in older people. Gerontology 1996; 42: 199-203.

45. Buatois S, Gueguen R, Gauchard GC, Benetos A, Perrin P. Posturography and risk of recurrent falls in healthy non-institutionalized persons aged over 65. Gerontology 2006; 52: 345-352.

46. Horak FB, Dickstein R, Peterka RJ. Diabetic neuropathy and surface swayreferencing disrupt somatosensory information for postural stability in stance. Somatosens Mot Res 2002; 19: 316-326.

47. Fox ZG, Mihalik JP, Blackburn JT, Battaglini CL, Guskiewicz KM. Return of postural control to baseline after anaerobic and aerobic exercise protocols. J Athl Train 2008; 43: 456 - 463.

48. Sparto PJ, Redfern MS. Quantification of direction and magnitude of cyclical postural sway using ellipses. Biomed Eng - Appl Basis Commun 2001; 13: 2137.

49. Lord SR, Menz HB. Visual contributions to postural stability in older adults. Gerontology. 2000; 46: $306-310$. 
50. Chen E, Fu A, Chan K, Tsang W. Balance control in very old adults with and without visual impairment. European Journal Of Applied Physiology May 2012; 112:1631-1636.

51. Nakamura, H, Tsuchida, T, Mano, Y. The assessment of posture control in the elderly using the displacement of the center of pressure after forward platform translation. J Electromyogr Kinesiol 2001; 11: 395- 403.

52. McCrory J, Chambers A, Daftary A, Redfern M. Dynamic postural stability in pregnant fallers and non-fallers. BJOG 2010; 117: 954-962.

53. McCrory JL, Chambers AJ, Daftary A, Redfern MS. Dynamic postural stability during advancing pregnancy. J Biomech. 2010; 43: 2434-9.

54. Hulme, J. A. Beyond kegels book ii. Missoula: Phoenix Publishing; 1998.

55. Leitner C, Mair P, Paul B, Wick F, Mittermaier C, Sycha T, Ebenbichler G. Reliability of posturohraphic measurements in the assessment of impaired sensorimotor function in chronic low back pain. Journal of Electromyography and Kinesiology. 2009; 19: 380-390.

56. Mihalik JP, Kohli L, Whitton MC. Do the physical characteristics of a virtual reality device contraindicate its use for balance assessment? J Sport Rehabil 2008; 17:38-49.

57. Sokal RR, Rohlf FJ. Biometry: The Principles and Practice of Statistics in Biological Research, 3rd edn. New York: W.H. Freeman and Company; 1995.

58. Ho TJ, Chen SC, Hong SW, Lu TW, Lin JG. Influence of long-term tai-chi chuan training on standing balance in the elderly. Biomedical Engineering: Applications, Basic and Communications 2012; 24: 15-25.

59. Pedalini MEB, Cruz OLM, Bittar RSM, Lorenzi MC, Grasel SS. Sensory organization test in elderly patients with and without vestibular dysfunction. Acta Oto-Laryngologica 2009; 129: 962-965.

60. Yoo EJ, Jun TW, Hawkins SA. The effects of a walking exercise program on fallrelated fitness, bone metabolism, and fall-related psychological factors in elderly women. Research in Sports Medicine. 2010; 18:236-250.

61. Wolfson L, Whipple R, Derby C, Judge J, King M, Amerman P, Schmidt J, Smyers $D$. Balance and strength training in older adults: intervention gains and tai chi maintenance. Journal of the American Geriatric Society 1996; 44:498-506. 
62. Talkowski JB, Brach JS, Studenski S, Newman AB. Impact of health perception, balance perception, fall history, balance performance, and gait speed on walking activity in older adults. Physical Therapy 2008; 88: 1474-1481.

63. Talley KMC, Wyman JF, Gross CR. Psychometric Properties of the ActivitiesSpecific Balance Confidence Scale and the Survey of Activities and Fear of Falling in Older Women. Journal of the American Geriatrics Society. 2008; 56: 328-333.

64. Liaw, MY, Chen, CL, Pei, YC, Leong, CP, \& Lau, YC. Comparison of the static and dynamic balance performance in young, middle-aged, and elderly healthy people. Chang Gung Medical Journal 2008; 32: 297-304.

65. Grace Gaerlan M, Alpert PT, Cross C, Louis M, Kowalski S. Postural balance in young adults: the role of visual, vestibular and somatosensory systems. Journal of the American Academy of Nurse Practitioners. 2012; 24: 375-381.

66. Asaka T, Yahata K, Mani H, Wang Y. Modulations of muscle modes in automatic postural responses induced by external surface translations. Journal of Motor Behavior 2011; 43: 165-172.

67. Davis DS, Mancinelli CA, Petronis JJ, Bensenhaver C, McClintic T, Nelson G. Variables associated with level of disability in working individuals with nonacute low back pain: a cross-sectional investigation. Journal of Orthopaedic \& Sports Physical Therapy. 2013; 43: 97-104.

68. Shumway-Cook A, Woollacott MH. Motor control, 2nd edn. Baltimore: Lippincott Williams \& Wilkins, 2001. pp 174.

69. Winters DA. Biomechanics and motor control of human movement, 2nd edn. New York: John Wiley \& Sons, Inc, 1990. pp 85-96.

70. Kuo AD, Zajac FE. Chapter 31 Human standing postural: multi-joint movement strategies based on biomechanical constraints. Progress in Brain Research. 1993; 97: 349-358.

71. Winter DA. Human balance and posture control during standing and walking. Gait \& Posture. 1995; 3: 193-214. 